\title{
The communication complexity of non-signaling distributions
}

\author{
Julien Degorre * $\quad$ Marc Kaplan ${ }^{\dagger} \quad$ Sophie Laplante ${ }^{\dagger} \quad$ Jérémie Roland $\ddagger$
}

\begin{abstract}
We study a model of communication complexity that encompasses many well-studied problems, including classical and quantum communication complexity, the complexity of simulating distributions arising from bipartite measurements of shared quantum states, and XOR games. In this model, Alice gets an input $x$, Bob gets an input $y$, and their goal is to each produce an output $a, b$ distributed according to some pre-specified joint distribution $p(a, b \mid x, y)$. Our results apply to any non-signaling distribution, that is, those where Alice's marginal distribution does not depend on Bob's input, and vice versa, therefore our techniques apply to any communication problem that can be reduced to a non-signaling distribution, including quantum distributions, Boolean and non-Boolean functions, most relations, partial (promise) problems, in the two-player and multipartite settings.

We give elementary proofs and very intuitive interpretations of the recent lower bounds of Linial and Shraibman, which we generalize to the problem of simulating any non-signaling distribution. The lower bounds we obtain are also expressed as linear programs (or SDPs for quantum communication). We show that the dual formulations have a striking interpretation, since they coincide with maximum violations of Bell and Tsirelson inequalities. The dual expressions are closely related to the winning probability of XOR games.

We show that as in the case of Boolean functions, the gap between the quantum and classical lower bounds is at most linear in size of the support of the distribution, and does not depend on the size of the inputs. This tranlates into a bound on the gap between maximal Bell and Tsirelson inequalities, which was previously known only for the case of Boolean outcomes with uniform marginals.

Finally, we give an exponential upper bound on quantum and classical communication complexity in the simultaneous messages model, for any non-signaling distribution. One consequence of this is a simple proof that any quantum distribution can be approximated with a constant number of bits of communication.
\end{abstract}

\section{Introduction}

Communication complexity of Boolean functions has a long and rich past, stemming from the paper of Yao in 1979 [Yao79], whose motivation was to study the area of VLSI circuits. In the years that followed, tremendous progress has been made in developing a rich array of lower bound techniques for various models of communication complexity (see e.g. [KN97]).

From the physics side, the question of studying how much communication is needed to simulate distributions arising from physical phenomena, such as measuring bipartite quantum states was posed in 1992 by Maudlin, a philosopher of science, who wanted to quantify the non-locality inherent to these systems [Mau92]. Maudlin, and the authors who followed [BCT99, Ste00, TB03, CGMP05, DLR07] (some independently of his work, and of each other) progressively improved upper bounds on simulating correlations of the 2 qubit singlet state. In a recent breakthrough, Regev and Toner [RT07] proved that two bits of communication suffice to simulate the correlations arising from two-outcome measurements of arbitrary-dimension bipartite quantum states.

In this paper, we consider the more general framework of simulating non-signaling distributions. These are distributions of the form $p(a, b \mid x, y)$, where Alice gets input $x$ and produces an output $a$, and Bob gets input $y$ and outputs $b$. The non-signaling condition is a fundamental property of bipartite physical systems, which states that the players gain no information on the other player's input. Distributions arising from quantum measurements on shared bipartite

\footnotetext{
${ }^{*}$ CNRS, Laboratoire Informatique de Grenoble

${ }^{\dagger}$ LRI, Université Paris-Sud

${ }^{\ddagger}$ NEC Laboratories America
} 
states are non-signaling, and the Boolean functions correspond to extremal non-signaling distributions with Boolean outcomes and uniform marginals.

Our main contribution is a new method for handling non-signaling distributions, based on affine combinations of lower-complexity distributions, which we apply for upper and lower bounds on communication complexity. Although they are formulated, and proven, in quite a different way, our lower bounds coincide with Linial and Shraibman's factorization norm lower bounds [LS08b] in the restricted case of Boolean functions. Similarly, our upper bounds extend the upper bounds of Shi and Zhu for approximating quantum distributions [SZ08] to all non-signaling distributions. All of our proofs use elementary techniques only, and the intuition they provide is remarkably simple.

When we consider the dual of our lower bounds, these turn out to correspond precisely to maximal Bell inequality violations in the case of classical communication, and Tsirelson inequality violations for quantum communication. Hence, we have made formal the intuition that large Bell inequalities should lead to large lower bounds on communication complexity.

We also show that there cannot be a large gap between these quantities. This was previously known only in the case of Boolean outcomes with uniform marginals, and followed by Tsirelson's theorem and Grothendieck's inequality, neither of which are known to extend beyond this special case. This also shows that our method, as was already the case for Linial and Shraibman's bounds, cannot hope to prove large gaps between classical and quantum communication complexity. While this is a negative result, it also sheds some light on the relationship between the Linial and Shraibman family of lower bound techniques, and the information theoretic methods, such as the recent subdistribution bound [JKN08], one of the few remaining lower bound techniques not known to follow from Linial and Shraibman. We give an example of a problem [BCT99] for which rectangle size gives an exponentially better lower bound than our method.

Summary of results The paper is organized as follows. In Section 2, we give the required definitions and models of communication complexity and characterizations of the classes of distributions we consider.

In Section 3, we prove our lower bound on classical and quantum communication, and show that it coincides with Linial and Shraibman's method in the special case of Boolean functions (Theorem 13).

Our lower bounds are linear programs (respectively, SDPs in the quantum case), and in Section 4 , we show that the dual linear programs (resp. SDPs) have a natural interpretation in quantum information, as they coincide with Bell (resp. Tsirelson) inequality violations (Theorem 17). We also give a dual expression which also has a natural interpretation, as the maximum winning probability of an associated XOR game (Corollary 19). The primal form is also the multiplicative inverse of the maximum winning probability of the associated XOR game, where all inputs have the same winning probability.

In Section 5, we compare the two methods and show that the quantum and classical lower bound expressions can differ by at most a factor that is linear in the number of outcomes. (Theorem 22).

Finally, in Section 6, we give upper bounds on simultaneous messages complexity in terms of our lower bound expression (Theorem 26). We use fingerprinting methods [BCWd01, Yao03, SZ08, GKd06] to give very simple proofs that classical communication with shared randomness, or quantum communication with shared entanglement, can be simulated in the simultaneous messages model, with exponential blowup in communication, and in particular that any quantum distribution can be approximated with constant communication.

Related work The use of affine combinations for non-signaling distributions has roots in the quantum logic community, where quantum non-locality has been studied within the setting of more general probability theories [FR81, RF81, KRF87, Wil92]. Until recently, this line of work was largely unknown in the quantum information theory community [Bar07, BBLW07].

The structure of the non-signaling polytope has been the object of much study. A complete characterization of the vertices has been obtained in some, but not all cases: for two players, the case of binary inputs [BLM $\left.{ }^{+} 05\right]$, and the case of binary outputs [BP05, JM05] are known, and for $n$ players, the case of Boolean inputs and outputs is known [BP05].

The work on simulating quantum distributions has focused mainly on providing upper bounds, and most results apply to simulating the correlations only. A few results address the simulation of quantum correlations with non-uniform marginals. Bacon and Toner give an upper bound of 2 bits for non-maximally entangled qubit pairs [TB03]. Shi and 
Zhu [SZ08] show a constant upper bound for approximating any quantum distribution (including the marginals) to within a constant.

Pironio gives a general lower bound technique based on Bell-like inequalities [Pir03]. There are a few ad hoc lower bounds on simulating quantum distributions, including a linear lower bound for a distribution based on Deutsch-Jozsa's problem [BCT99], and a recent lower bound of Gavinsky [Gav08].

The $\gamma_{2}$ method was first introduced as a measure of the complexity of matrices [LMSS07]. It was shown to be a lower bound on communication complexity [LS08b], and to generalize many previously known methods. Lee et al. use it to establish direct product theorems and relate the dual norm of $\gamma_{2}$ to the value of XOR games [LSŠ08]. Lee and Shraibman [LS08a] use a multidimensional generalization of a related quantity $\mu$ (where the norm-1 ball consists of cylinder intersections) to prove a lower bound in the multiparty number-on-the-forehead-model, for the disjointness function.

\section{Preliminaries}

In this paper, we extend the framework of communication complexity to non-signaling distributions. This framework encompasses the standard models of communication complexity of Boolean functions but also total and partial nonBoolean functions and relations, as well as distributions arising from the measurements of bipartite quantum states. Most results we present also extend to the multipartite setting.

\subsection{Non-signaling distributions}

Non-signaling, a fundamental postulate of physics, states that any observation on part of a system cannot instantaneously affect a remote part of the system, or similarly, that no signal can travel instantaneously. We consider distributions $p(a, b \mid x, y)$ where $x \in \mathcal{X}, y \in \mathcal{Y}$ are the inputs of the players, and they are required to each produce an outcome $a \in \mathcal{A}, b \in \mathcal{B}$, distributed according to $p(a, b \mid x, y)$. We restrict ourselves to the distributions where each player's outcome does not depend on the other player's input. Mathematically, non-signaling (also called causality) is defined as follows.

Definition 1 (Non-signaling distributions). A bipartite, conditional distribution $\mathbf{p}$ is non-signaling if

$$
\begin{aligned}
& \forall a, x, y, y^{\prime}, \quad \sum_{b} p(a, b \mid x, y)=\sum_{b} p\left(a, b \mid x, y^{\prime}\right), \\
& \forall b, x, x^{\prime}, y, \quad \sum_{a} p(a, b \mid x, y)=\sum_{a} p\left(a, b \mid x^{\prime}, y\right) .
\end{aligned}
$$

For any non-signaling distribution, the marginal distribution on Alice's output $p(a \mid x, y)=\sum_{b} p(a, b \mid x, y)$ does not depend on $y$, so we write $p(a \mid x)$, and similarly $p(b \mid y)$ for the marginal distribution on Bob's output. We denote by $\mathcal{C}$ the set of all non-signaling distributions.

In the case of binary outcomes, more specifically, $\mathcal{A}=\mathcal{B}=\{ \pm 1\}$, it is known that a non-signaling distribution is uniquely determined by the (expected) correlations, defined as $C(x, y)=E(a \cdot b \mid x, y)$, and the (expected) marginals, defined as $M_{A}(x)=E(a \mid x), M_{B}(y)=E(b \mid y)$.

Proposition 1. For any functions $C: \mathcal{X} \times \mathcal{Y} \rightarrow[-1,1], M_{A}: \mathcal{X} \rightarrow[-1,1], M_{B}: \mathcal{Y} \rightarrow[-1,1]$, satisfying $1+a \cdot b C(x, y)+a M_{A}(x)+b M_{B}(y) \geq 0 \forall(x, y) \in \mathcal{X} \times \mathcal{Y}$ and $a, b \in\{ \pm 1\}$, there is a unique non-signaling distribution $\mathbf{p}$ such that $\forall x, y, E(a \cdot b \mid x, y)=C(x, y)$ and $E(a \mid x)=M_{A}(x)$ and $E(b \mid y)=M_{B}(y)$, where $a, b$ are distributed according to $\mathbf{p}$.

Proof. Fix $x, y . C, M_{A}, M_{B}$ are obtained from $\mathbf{p}$ by the following full rank system of equations.

$$
\left(\begin{array}{rrrr}
1 & -1 & -1 & 1 \\
1 & 1 & -1 & -1 \\
1 & -1 & 1 & -1 \\
1 & 1 & 1 & 1
\end{array}\right)\left(\begin{array}{c}
p(+1,+1 \mid x, y) \\
p(+1,-1 \mid x, y) \\
p(-1,+1 \mid x, y) \\
p(-1,-1 \mid x, y)
\end{array}\right)=\left(\begin{array}{c}
C(x, y) \\
M_{A}(x) \\
M_{B}(y) \\
1
\end{array}\right)
$$

Computing the inverse yields $p(a, b \mid x, y)=\frac{1}{4}\left(1+a \cdot b C(x, y)+a M_{A}(x)+b M_{B}(y)\right)$. 
We will write $\mathbf{p}=\left(C, M_{A}, M_{B}\right)$ and use both notations interchangeably when considering distributions over binary outcomes. We also denote by $\mathcal{C}_{0}$ the set of non-signaling distributions with uniform marginals, that is, $\mathbf{p}=$ $(C, 0,0)$, and write $C \in \mathcal{C}_{0}$, omitting the marginals when there is no ambiguity.

\subsubsection{Boolean functions}

The communication complexity of Boolean functions is a special case of the problem of simulating non-signaling distributions. As we shall see in Section 2.3. it happens that the associated distributions are extremal points of the non-signaling polytope. If the distribution stipulates that the product of the players' outputs equal some function $f: \mathcal{X} \times \mathcal{Y} \rightarrow\{ \pm 1\}$ then this corresponds to the standard model of communication complexity (up to an additional bit of communication, for Bob to output $f(x, y)$ ). If we further require that Alice's output be +1 or -1 with equal probability, likewise for Bob, then the distribution is non-signaling and has the following form:

Definition 2. For a function $f: \mathcal{X} \times \mathcal{Y} \rightarrow\{-1,1\}$, denote $\mathbf{p}_{f}$ the distribution defined by $p_{f}(a, b \mid x, y)=\frac{1}{2}$ if $f(x, y)=a \cdot b$ and 0 otherwise. Equivalently, $\mathbf{p}_{f}=\left(C_{f}, 0,0\right)$ where $C_{f}(x, y)=f(x, y)$.

In the case of randomized communication complexity, a protocol that simulates a Boolean function with error probability $\epsilon$ corresponds to simulating correlations $C^{\prime}$ scaled down by a factor at most $1-2 \epsilon$, that is, $\forall x, y, \operatorname{sgn}\left(C^{\prime}(x, y)\right)=$ $C_{f}(x, y)$ and $\left|C^{\prime}(x, y)\right| \geq 1-2 \epsilon$. While we will not consider these cases in full detail, non-Boolean functions, partial functions and some classes of relations may be handled in a similar fashion, hence our techniques can be used to show lower bounds in these settings as well.

\subsubsection{Quantum distributions}

Of particular interest in the study of quantum non-locality are the distributions arising from measuring bipartite quantum states. We will use the following definition:

Definition 3. A distribution $\mathbf{p}$ is quantum if there exists a quantum state $|\psi\rangle$ in a Hilbert space $\mathcal{H}$ and measurement operators $\left\{E_{a}(x): a \in \mathcal{A}, x \in \mathcal{X}\right\}$ and $\left\{E_{b}(y): b \in \mathcal{B}, y \in \mathcal{Y}\right\}$, such that $p(a, b \mid x, y)=\left\langle\psi\left|E_{a}(x) E_{b}(y)\right| \psi\right\rangle$, with the measurement operators satisfying

1. $E_{a}(x)^{\dagger}=E_{a}(x)$ and $E_{b}(y)^{\dagger}=E_{b}(y)$,

2. $E_{a}(x) \cdot E_{a^{\prime}}(x)=\delta_{a a^{\prime}} E_{a}(x)$ and $E_{b}(y) \cdot E_{b^{\prime}}(y)=\delta_{b b^{\prime}} E_{b}(y)$,

3. $\sum_{a} E_{a}(x)=\mathbb{1}$ and $\sum_{b} E_{b}(x)=\mathbb{1}$, where $\mathbb{1}$ is the identity operators on $\mathcal{H}$,

4. $E_{a}(x) \cdot E_{b}(y)=E_{b}(y) \cdot E_{a}(x)$.

Note that a more standard definition would be to replace the last condition on the measurement operators (commutativity) by the stronger condition that the operators $E_{a}(x)$ act non-trivially on a subspace $\mathcal{H}_{A}$ only, and that the operators $E_{b}(y)$ act non-trivially on a subspace $\mathcal{H}_{B}$ only, with $\mathcal{H}=\mathcal{H}_{A} \otimes \mathcal{H}_{B}$. If we restrict the Hilbert space $\mathcal{H}$ to be finite-dimensional, these two definitions are equivalent, but whether this also holds in full generality is still unknown. We use this less standard definition because this will allow us to use the results from [NPA08] (see this reference for a discussion about the different definitions).

We denote by $\mathcal{Q}$ the set of all quantum distributions. In the restricted case of binary outcomes with uniform marginals, we let $\mathcal{Q}_{0}$ be the set of all quantum correlations.

The communication complexity of simulating traceless binary measurements on maximally entangled states has been settled by Regev and Toner using two bits of communication, since in this case the marginals are uniform [RT07]. Their technique also handles general binary measurements on any entangled state, but in this case they only simulate the correlations. The complexity of simulating the full joint distribution exactly when the marginals are non-uniform remains open. 


\subsection{Models of communication complexity}

We consider the following model of communication complexity of non-signaling distributions $\mathbf{p}$. Alice gets input $x$, Bob gets input $y$, and after exchanging bits or qubits, Alice has to output $a$ and Bob $b$ so that the joint distribution is $p(a, b \mid x, y)$. In the classical setting we allow shared randomness, and in the quantum setting we allow shared entanglement. When needed, we use superscripts $p u b$ and ent to denote shared randomness and quantum entanglement. $R_{0}(\mathbf{p})$ denotes the communication complexity of simulating $\mathbf{p}$ exactly, using shared randomness and classical communication. $Q_{0}(\mathbf{p})$ denotes the communication complexity of simulating $\mathbf{p}$ exactly, using shared entanglement and quantum communication. For $R_{\epsilon}(\mathbf{p})$, we are only required to simulate some distribution $\mathbf{p}^{\prime}$ such that $\delta\left(\mathbf{p}, \mathbf{p}^{\prime}\right) \leq \epsilon$, where $\delta\left(\mathbf{p}, \mathbf{p}^{\prime}\right)=\max \left\{\left|p(\mathcal{E} \mid x, y)-p^{\prime}(\mathcal{E} \mid x, y)\right|: x, y \in \mathcal{X} \times \mathcal{Y}, \mathcal{E} \subseteq \mathcal{A} \times \mathcal{B}\right\}$ is the total variation distance (or statistical distance) between two distributions.

For distributions with binary outcomes, we write $R_{\epsilon}\left(C, M_{A}, M_{B}\right), Q_{\epsilon}\left(C, M_{A}, M_{B}\right)$. In the case of Boolean functions, $R_{\epsilon}(C)=R_{\epsilon}(C, 0,0)$ corresponds to the usual notion of computing $f$ with probability at least $1-\epsilon$, where $C$ is the \pm 1 communication matrix of $f$.

From the point of view of communication, distributions with uniform marginals are the easiest to simulate. Suppose we have a protocol that simulates correlations $C$ with arbitrary marginals. By using just an additional shared random bit, both players can flip their outcome whenever the shared random bit is 1 . Since each players' marginal outcome is now an even coin flip, this protocol simulates the distribution $(C, 0,0)$.

Proposition 2. For any Boolean non-signaling distribution $\left(C, M_{A}, M_{B}\right)$, simulating $(C, 0,0)$ requires no more communication than simulating $\left(C, M_{A}, M_{B}\right)$ (with 1 additional bit of shared randomness).

\subsection{Characterization of the sets of local and non-signaling distributions}

In the quantum information literature, the distributions that can be simulated with shared randomness and no communication (also called a local hidden variable model) are called local distributions.

Definition 4. Local deterministic distributions are of the form $p(a, b \mid x, y)=\delta_{a=\lambda_{A}(x)} \cdot \delta_{b=\lambda_{B}(y)}$ where $\lambda_{A}: \mathcal{X} \rightarrow \mathcal{A}$ and $\lambda_{B}: \mathcal{Y} \rightarrow \mathcal{B}$, and $\delta$ is the Kronecker delta. A distribution is local if it can be written as a convex combination of local deterministic distributions.

We denote by $\Lambda$ the set of local deterministic distributions $\left\{\mathbf{p}^{\lambda}\right\}_{\lambda \in \Lambda}$ and by $\mathcal{L}$ the set of local distributions. Let $\operatorname{conv}(A)$ denote the convex hull of $A$. In the case of binary outcomes, we have

Proposition 3. $\mathcal{L}=\operatorname{conv}\left(\left\{\left(u^{T} v, u, v\right): u \in\{ \pm 1\}^{\mathcal{X}}, v \in\{ \pm 1\}^{\mathcal{Y}}\right\}\right)$.

We also denote by $\mathcal{L}_{0}$ the set of local correlations over binary outcomes with uniform marginals.

The quantum information literature reveals a great deal of insight into the structure of the classical, quantum, and non-signaling distributions. It is well known that $\mathcal{L}$ and $\mathcal{C}$ are polytopes. While the extremal points of $\mathcal{L}$ are simply the local deterministic distributions, the non-signaling polytope $\mathcal{C}$ has a more complex structure [JM05, BP05]. $\mathcal{C}_{0}$ is the convex hull of the distributions obtained from Boolean functions.

Proposition 4. $\mathcal{C}_{0}=\operatorname{conv}\left(\left\{\left(C_{f}, 0,0\right): C_{f} \in\{ \pm 1\}^{\mathcal{X} \times \mathcal{Y}}\right\}\right)$.

We show that $\mathcal{C}$ is the affine hull of the local polytope (restricted to the positive orthant since all probabilities $p(a, b \mid x, y)$ must be positive). We give a simple proof for the case of binary outcomes but this carries over to the general case. This was shown independently of us, on a few occasions in different communities [RF81, FR81, KRF87, Wil92, Bar07].

Theorem 5. $\mathcal{C}=\operatorname{aff}^{+}\{\mathcal{L}\}$, where $\operatorname{aff}^{+}\{\mathcal{L}\}$ is the restriction to the positive orthant of the affine hull of $\mathcal{L}$, and $\operatorname{dim} \mathcal{C}=\operatorname{dim} \mathcal{L}=|\mathcal{X}| \times|\mathcal{Y}|+|\mathcal{X}|+|\mathcal{Y}|$.

Proof. We show that $\operatorname{aff}(\mathcal{C})=\operatorname{aff}(\mathcal{L})$. The theorem then follows by restricting to the positive orthant, and using the fact that $\mathcal{C}=\operatorname{aff}^{+}(\mathcal{C})$.

$\operatorname{aff}(\mathcal{L}) \subseteq \operatorname{aff}(\mathcal{C})]$ Since any local distribution satisfies the (linear) non-signaling constraints in Def. 4, this is also true for any affine combination of local distributions. 
[aff $(\mathcal{C}) \subseteq \operatorname{aff}(\mathcal{L})]$ For any $(\sigma, \pi) \in \mathcal{X} \times \mathcal{Y}$, we define the distribution $\mathbf{p}_{\sigma \pi}=\left(C_{\sigma \pi}, u_{\sigma \pi}, v_{\sigma \pi}\right)$ with correlations $C_{\sigma \pi}(x, y)=\delta_{x=\sigma} \delta_{y=\pi}$ and marginals $u_{\sigma \pi}(x)=0, v_{\sigma \pi}(y)=0$. Similarly, we define for any $\sigma \in \mathcal{X}$ the distribution $\mathbf{p}_{\sigma .}=\left(C_{\sigma .}, u_{\sigma .}, v_{\sigma}.\right)$ with $C_{\sigma \cdot} \cdot(x, y)=0, u_{\sigma} .(x)=\delta_{x=\sigma}, v_{\sigma} \cdot(y)=0$, and for any $\pi \in \mathcal{Y}$ the distribution $\mathbf{p}_{\cdot \pi}=$ $\left(C \cdot \pi, u_{\cdot \pi}, v_{\cdot \pi}\right)$ with $C_{\cdot \pi}(x, y)=0, u_{\cdot \pi}(x)=0, v_{\cdot \pi}(y)=\delta_{y=\pi}$. It is straightforward to check that these $|\mathcal{X}| \times|\mathcal{Y}|+$ $|\mathcal{X}|+|\mathcal{Y}|$ distributions are local, and that they constitute a basis for the vector space embedding aff $(\mathcal{C})$, which consists of vectors of the form $(C, u, v)$.

This implies that while local distributions are convex combinations of local deterministic distributions $\mathbf{p}^{\lambda} \in \Lambda$, non-signaling distributions are affine combinations of these distributions.

Corollary 6 (Affine model). A distribution $\mathbf{p} \in \mathcal{C}$ if and only if $\exists q_{\lambda} \in \mathbb{R}$ with $\mathbf{p}=\sum_{\lambda \in \Lambda} q_{\lambda} \mathbf{p}^{\lambda}$.

Note that since $\mathbf{p}$ is a distribution, this implies $\sum_{\lambda \in \Lambda} q_{\lambda}=1$. Since weights in an affine combination may be negative, but still sum up to one, this may be interpreted as a quasi-mixture of local distributions, some distributions being used with possibly "negative probability". Surprisingly this is not a new notion; see for example Groenewold [Gro85] who gave an affine model for quantum distributions; or a discussion of "negative probability" by Feynman [Fey86].

\subsection{Characterization of the set of quantum distributions}

As for the set of quantum distributions $\mathcal{Q}$, it is known to be convex, but not a polytope. Although no simple characterization of $\mathcal{Q}$ is known, Navascues, Pironio and Acin have given a characterization for a hierarchy of sets $\left\{\mathcal{Q}^{n}: n \in \mathbb{N}_{0}\right\}$, such that $\mathcal{Q}^{n} \subseteq \mathcal{Q}^{n-1}$ and $\mathcal{Q}^{n} \rightarrow \mathcal{Q}$ for $n \rightarrow \infty$ [NPA08]. We briefly introduce this hierarchy because it will be useful in Section 4] but we refer the reader to [NPA08] for full details.

Let $\mathcal{S}_{n}$ be the set of all monomials of degree up to $n$ in measurement operators $E_{a}(x)$ and $E_{b}(y)$ (for example, $\mathbb{1}, E_{a}(x)$ and $E_{a}(x) E_{a^{\prime}}(x) E_{b}(y)$ are a monomials of degree 0,1 and 3, respectively). Due to the conditions in Definition 3 , the operators in $\mathcal{S}_{n}$ (and their Hermitian conjugates) satisfy linear equations such as $E_{a}(x)^{\dagger}-E_{a}(x)=0$, $\sum_{a} E_{a}(x)-\mathbb{1}=0$, or higher order equations such as $E_{a}(x)^{\dagger} E_{a}(x) E_{b}(y)-E_{b}(y)^{\dagger} E_{a}(x)=0$. Let us suppose that we have $m(n)$ linearly independent equations for the operators in $\mathcal{S}_{n}$. These equations may be written as $\sum_{S, T \in \mathcal{S}_{n}}\left(F_{k}\right)_{S, T} S^{\dagger} T=0$, where, for all $k \in[m(n)], F_{k}$ is a matrix whose rows and columns are labelled by the elements of $\mathcal{S}_{n}$. We are now ready to define the set of distributions $\mathcal{Q}^{n}$.

Definition 5 (Quantum hierarchy). A distribution $\mathbf{p}$ is in $\mathcal{Q}^{n}$ if and only if there exists a positive-semidefinite matrix $\Gamma \succcurlyeq 0$, whose rows and columns are labelled by the elements of $\mathcal{S}_{n}$, satisfying

1. $\Gamma_{\mathbb{1}, \mathbb{1}}=1$,

2. $\Gamma_{E_{a}(x), E_{b}(y)}=p(a, b \mid x, y)$, for all $a, b, x, y \in \mathcal{A} \times \mathcal{B} \times \mathcal{X} \times \mathcal{Y}$

3. $\operatorname{tr}\left(F_{k}^{\dagger} \Gamma\right)=0$ for all $k \in[m(n)]$.

Comparing with Definition 3 for $\mathcal{Q}$, we immediately get that $\mathcal{Q} \subseteq \mathcal{Q}^{n}$ by setting $\Gamma_{S, T}=\left\langle\psi\left|S^{\dagger} T\right| \psi\right\rangle$. The proof that $\mathcal{Q}^{n}$ converges to $\mathcal{Q}$ is much more involved and is given in [NPA08].

In the special case of binary outcomes with uniform marginals, the hierarchy collapses at the first level, that is, $\mathcal{Q}_{0}^{1}=\mathcal{Q}_{0}$. This was known before the hierarchy was introduced, as a consequence of the following theorem of Tsirelson.

Theorem 7 ([Tsi85]). Let $\mathbb{S}_{n}$ be the set of unit vectors in $\mathbb{R}^{n}$, and $\mathcal{H}^{d}$ be a d-dimensional Hilbert space.

1. If $\left(C, M_{A}, M_{B}\right) \in \mathcal{Q}$ is a probability distribution obtained by performing binary measurements on a quantum state $|\psi\rangle \in \mathcal{H}^{d} \otimes \mathcal{H}^{d}$, then there exists vectors $\vec{a}(x), \vec{b}(y) \in \mathbb{S}_{2 d^{2}}$ such that $C(x, y)=\vec{a}(x) \cdot \vec{b}(y)$.

2. If $\vec{a}(x), \vec{b}(y)$ are unit vectors in $\mathbb{S}_{n}$, then there exists a probability distribution $(C, 0,0) \in \mathcal{Q}$ obtained by performing binary measurements on a maximally entangled state $|\psi\rangle \in \mathcal{H}^{\left\lfloor\frac{n}{2}\right\rfloor} \otimes \mathcal{H}^{\left\lfloor\frac{n}{2}\right\rfloor}$ such that $C(x, y)=$ $\vec{a}(x) \cdot \vec{b}(y)$.

Corollary 8. $\mathcal{Q}_{0}=\{C: C(x, y)=\vec{a}(x) \cdot \vec{b}(y),\|\vec{a}(x)\|=\|\vec{b}(y)\|=1 \forall x, y\}$. 
Clearly, $\mathcal{L} \subseteq \mathcal{Q} \subseteq \mathcal{C}$. The existence of Grothendieck's constant (see e.g. [AN06]) implies the following statement.

Proposition 9. $\mathcal{L}_{0} \subseteq \mathcal{Q}_{0} \subseteq K_{G} \mathcal{L}_{0}$, where $K_{G}$ is Grothendieck's constant.

\section{Lower bounds for non-signaling distributions}

We extend Linial and Shraibman's factorization norm $\left(\gamma_{2}\right)$ and nuclear norm $(\nu)$ lower bound methods [LS08b] to the simulation of any non-signaling distributions. The proof we give is simple, especially in the setting studied by Linial and Shraibman, for Boolean functions, which corresponds in our setting to binary outputs and uniform marginal distributions. The main intuition is that $c$ bits of communication can increase correlations by at most a factor of $2^{c}$.

\subsection{Communication vs correlations}

We first show that if a distribution $\mathbf{p}$ may be simulated with $c$ bits of communication (or $q$ qubits of quantum communication), then a scaled-down version of this distribution is local (or quantum). From this local (or quantum) distribution, we derive an affine model for $\mathbf{p}$ (Theorem 13) which gives the lower bound on communication.

Definition 6 (Scaled-down distribution). Let $\mathbf{p}$ be a conditional probability distribution over $\mathcal{A} \times \mathcal{B}$, with $|\mathcal{A}|=A$ and $|\mathcal{B}|=B$. For $0 \leq \pi_{A}, \pi_{B} \leq 1$, we define the scaled-down probability distribution $\mathbf{p}_{\pi_{A}, \pi_{B}}$ as

$$
p_{\pi_{A}, \pi_{B}}(a, b \mid x, y)=\pi p(a, b \mid x, y)+\pi_{A}\left(1-\pi_{B}\right) p(a \mid x) \frac{1}{B}+\left(1-\pi_{A}\right) \pi_{B} \frac{1}{A} p(b \mid y)+\left(1-\pi_{A}\right)\left(1-\pi_{B}\right) \frac{1}{A B},
$$

where $\pi=\pi_{A} \cdot \pi_{B}$.

In the binary case $\mathbf{p}=\left(C, M_{A}, M_{B}\right)$, the scaled down distribution is $\mathbf{p}_{\pi_{A}, \pi_{B}}=\left(\pi C, \pi_{A} M_{A}, \pi_{B} M_{B}\right)$. For distributions $\mathbf{p}_{f}$ arising from Boolean functions, this corresponds to scaling down the success probability by a factor of $\pi$.

Theorem 10. 1. Let $\mathbf{p}$ be a distribution that may be simulated with $c$ bits of classical communication, $c_{A}$ from Alice to Bob and $c_{B}$ from Bob to Alice. Then, $\mathbf{p}_{\pi_{A}, \pi_{B}}$ is local for $\pi_{A}=2^{-c_{A}}, \pi_{B}=2^{-c_{B}}$.

2. Let $\mathbf{p}$ be a distribution that may be simulated with $q$ qubits of quantum communication, $q_{A}$ from Alice to Bob and $q_{B}$ from Bob to Alice. Then, $\mathbf{p}_{\pi_{A}, \pi_{B}}$ is quantum for $\pi_{A}=2^{-2 q_{A}}, \pi_{B}=2^{-2 q_{B}}$.

3. If $Q_{0}\left(C, M_{A}, M_{B}\right) \leq q$ then $\pi C \in \mathcal{Q}_{0}$ for $\pi=2^{-q}$.

Proof. Let $P$ be a protocol simulating $\mathbf{p}$ with classical communication and shared randomness $\lambda$. We show how to simulate $\mathbf{p}_{\pi_{A}, \pi_{B}}$ without any communication. To get the key property we need for the probability analysis, we start by cleaning up the protocol and defining what we mean by a valid transcript. We assume that from Alice's point of view, any bit she receives from Bob can be either a 0 or a 1, that is, for any $x$, there is some input $y$ where Bob sends 0 and some $y^{\prime}$ where Bob sends 1 . Any other bit of the transcript is called a useless bit, and this can be detected by both players, therefore can be removed from the protocol. We further need to assume that the direction in which the bits are sent does not depend on the inputs, so we add useless bits set to 1 to the protocol wherever necessary. Note that this increases the communication by at most a factor of 2 , for example, communication can be made to alternate between Alice and Bob.

Let $T \in\{0,1\}^{c}$ be a $c$-bit string, interpreted as the transcript of the communication between Alice and Bob. From $T$, let us isolate the substring $T_{A} \in\{0,1\}^{c_{A}}$ of bits communicated by Alice to Bob (for example, these could be the bits of $T$ with an odd index), and similarly the substring $T_{B} \in\{0,1\}^{c_{B}}$ of bits communicated by Bob to Alice. This is well-defined since the direction of the bits does not depend on the input $x, y$. We write $T=\left(T_{A}, T_{B}\right)$.

From Alice's point of view, for given $x, \lambda$, we say that a $c$-bit string $T$ is a valid transcript for Alice if there exists $y$ such that, when simulating the protocol on input $x, y$ and shared randomness $\lambda$, the transcript matches $T$ on all useful bits, and she checks that all useless bits she sends to Bob are set to 1. Alice ignores the useless bits sent by Bob.

Fix $x, y, \lambda$. The key property we need is that for any $T_{B}$, there is a unique $T_{A}$ such that $\left(T_{A}, T_{B}\right)$ is valid for Alice. That there is at most one $T_{A}$ follows from the fact that Alice's protocol is deterministic once $\lambda$ is fixed. That there is 
at least one follows from the fact that Alice accepts as valid any useless bits in the transcript. We will also need the fact that when $T$ is chosen at random, the events " $T$ is valid for Alice" and " $T$ is valid for Bob" are independent. This is because the probability that Alice accepts or rejects $T$ does not depend on $T_{B}$, and vice versa.

To simulate $\mathbf{p}_{\pi_{A}, \pi_{B}}$, on input $x, y$ and shared randomness $\lambda$, Alice and Bob use additional shared randomness to pick a random $c$-bit transcript $T$. Alice outputs as follows:

1. If $T$ is valid for Alice, she outputs according to $P$,

2. otherwise, she outputs $a \in \mathcal{A}$ according to the uniform distribution.

Bob's protocol is similar.

Recall that $x, y, \lambda$ are fixed. Since for every $T_{B}$, there is exactly one $T_{A}$ for which $\left(T_{A}, T_{B}\right)$ is valid for Alice, the event " $T$ is valid for Alice" occurs with probability $2^{c_{B}} \frac{1}{2^{c}}=2^{-c_{A}}$ when $T$ is chosen uniformly at random.

Let $\pi_{A}=2^{-c_{A}}, \pi_{B}=2^{-c_{B}}$ and $\pi=2^{-c}$. During a particular execution of the protocol, one out of these four possibilities may happen:

1. with probability $\pi, T$ is valid for both Alice and Bob, in which case we obtain the original probability distribution $p(a, b \mid x, y)$,

2. with probability $\pi_{A}\left(1-\pi_{B}\right)$, Alice's output has the right marginal $p(a \mid x)$, but Bob's output is just uniformly random, $p_{U}(b \mid y)=\frac{1}{B}$,

3. with probability $\left(1-\pi_{A}\right) \pi_{B}$, Bob's output has the right marginal $p(b \mid y)$, but Alice's output is just uniformly random, $p_{U}(a \mid x)=\frac{1}{A}$,

4. with probability $\left(1-\pi_{A}\right)\left(1-\pi_{B}\right)$, their outputs are uniformly random, $p_{U}(a, b \mid x, y)=\frac{1}{A B}$.

As a consequence, it is straightforward to check that $P^{\prime}$ simulates $\mathbf{p}_{\pi_{A}, \pi_{B}}$, so that $\mathbf{p}_{\pi_{A}, \pi_{B}} \in \mathcal{L}$ since $P^{\prime}$ uses shared randomness but no communication.

For quantum protocols, we first simulate quantum communication using shared entanglement and teleportation, which uses 2 bits of classical communication for each qubit. Starting with this protocol using $2 q$ bits of classical communication, we may use the same idea as in the classical case, that is choosing a random $2 q$-bit string interpreted as the transcript, and replacing the players' respective outputs by independent random bits if the random transcript does not match the bits they would have sent in the original protocol. It then follows that $\mathbf{p}_{\pi_{A}, \pi_{B}} \in \mathcal{Q}$ for $\pi_{A}=$ $2^{-2 q_{A}}, \pi_{B}=2^{-2 q_{B}}$.

In the case of binary outputs with uniform marginals, we may improve the exponent of the scaling-down coefficient $\pi$ by a factor of 2 by using a variation of a result by [Kre95, Ya093, LS08b] (the proof is given in Appendix $\mathrm{A}$ for completeness).

Lemma 11 ([Kre95, Yao93, LS08b]). Let $\left(C, M_{A}, M_{B}\right)$ be a distribution simulated by a quantum protocol with shared entanglement using $q_{A}$ qubits of communication from Alice to Bob and $q_{B}$ qubits from Bob to Alice. There exist vectors $\vec{a}(x), \vec{b}(y)$ with $\|\vec{a}(x)\| \leq 2^{q_{B}}$ and $\|\vec{b}(y)\| \leq 2^{q_{A}}$ such that $C(x, y)=\vec{a}(x) \cdot \vec{b}(y)$.

The fact that $\pi C \in \mathcal{Q}_{0}$ for $\pi=2^{-q}$ then follows from Theorem 7 part 2.

\subsection{Communication vs affine models}

By Theorem 5, we know that any non-signaling distribution can be written as an affine combination of local distributions, which we call affine model. In this section we show that using Theorem 10, an explicit affine model can be derived from a (classical or quantum) communication protocol for $\mathbf{p}$, which gives us a lower bound technique for communication complexity in terms of how "good" the affine model is.

Let us define the following quantities, which as we will see may be considered as extensions of the $\nu$ and $\gamma_{2}$ quantities of [LS08b] (defined below) to distributions.

Definition 7. $\quad \tilde{\nu}(\mathbf{p})=\min \left\{\sum_{i}\left|q_{i}\right|: \exists \mathbf{p}_{i} \in \mathcal{L}, q_{i} \in \mathbb{R}, \mathbf{p}=\sum_{i} q_{i} \mathbf{p}_{i}\right\}$,

- $\tilde{\gamma}_{2}(\mathbf{p})=\min \left\{\sum_{i}\left|q_{i}\right|: \exists \mathbf{p}_{i} \in \mathcal{Q}, q_{i} \in \mathbb{R}, \mathbf{p}=\sum_{i} q_{i} \mathbf{p}_{i}\right\}$, 
- $\tilde{\nu}^{\epsilon}(\mathbf{p})=\min \left\{\tilde{\nu}\left(\mathbf{p}^{\prime}\right): \delta\left(\mathbf{p}, \mathbf{p}^{\prime}\right) \leq \epsilon\right\}$

- $\tilde{\gamma}_{2}^{\epsilon}(\mathbf{p})=\min \left\{\tilde{\gamma}_{2}\left(\mathbf{p}^{\prime}\right): \delta\left(\mathbf{p}, \mathbf{p}^{\prime}\right) \leq \epsilon\right\}$

The quantities $\tilde{\nu}(\mathbf{p})$ and $\tilde{\gamma}_{2}(\mathbf{p})$ show how well $\mathbf{p}$ may be represented as an affine combination of local or quantum distributions, a good affine combination being one where the sum of absolute values of coefficients $q_{i}$ is as low as possible. For a local distribution, we may take positive coefficients $q_{i}$, and therefore obtain the minimum possible value $\tilde{\nu}(\mathbf{p})=1$ (note that $\sum_{i} q_{i} \mathbf{p}_{i}=\mathbf{p}$ implies in particular $\sum_{i} q_{i}=1$ ), and similarly for quantum distributions, so that

Lemma 12. $\mathbf{p} \in \mathcal{L} \Longleftrightarrow \tilde{\nu}(\mathbf{p})=1$, and $\mathbf{p} \in \mathcal{Q} \Longleftrightarrow \tilde{\gamma}_{2}(\mathbf{p})=1$.

In other words, the set of local distributions $\mathcal{L}$ form the unit sphere of $\tilde{\nu}$, and similarly the set of quantum distributions $\mathcal{Q}$ form the unit sphere of $\tilde{\gamma}_{2}$. In the binary case, observe that by Proposition 2 , we have $\tilde{\gamma}_{2}(C) \leq \tilde{\gamma}_{2}(C, u, v)$ and $\tilde{\nu}(C) \leq \tilde{\nu}(C, u, v)$. By Proposition $9 \tilde{\gamma}_{2}(C) \leq \tilde{\nu}(C) \leq K_{G} \tilde{\gamma}_{2}(C)$. Similar properties hold for the approximate versions $\tilde{\nu}^{\epsilon}(C)$ and $\tilde{\gamma}_{2}^{\epsilon}(C)$.

We have shown (Theorem 10) that distributions scaled down exponentially in the communication are local; from these local protocols we can build up an affine model for the original distribution, in order to establish the lower bound.

Theorem 13. Let $\mathbf{p}$ be a conditional non-signaling distribution over $\mathcal{A} \times \mathcal{B}$, with $|\mathcal{A}|=A$ and $|\mathcal{B}|=B$.

1. If $R_{0}(\mathbf{p}) \leq c$, then $\tilde{\nu}(\mathbf{p}) \leq 2^{c+1}-1$. When $A=B=2, \tilde{\nu}(\mathbf{p}) \leq 2^{c}$.

2. If $Q_{0}(\mathbf{p}) \leq q$, then $\tilde{\gamma}_{2}(\mathbf{p}) \leq 2^{2 q+1}-1$. When $A=B=2, \tilde{\gamma}_{2}(\mathbf{p}) \leq 2^{2 q}$.

3. If $Q_{0}\left(C, M_{A}, M_{B}\right) \leq q$, then $\tilde{\gamma}_{2}(C, 0,0) \leq 2^{q}$.

Proof. We give a proof for the classical case, the quantum case follows by using teleportation. Let $c_{A}$ be the number of bits sent from Alice to Bob and $c_{B}$ from Bob to Alice. From Theorem 10, we know that $\mathbf{p}_{\pi_{A}, \pi_{B}}$ is local for $\pi_{A}=2^{-c_{A}}, \pi_{B}=2^{-c_{B}}$. Let $P^{\prime}$ be a protocol for $\mathbf{p}_{\pi_{A}, \pi_{B}}$, using shared randomness and no communication. Notice that

$$
p(a, b \mid x, y)=\frac{1}{\pi} p_{\pi_{A}, \pi_{B}}(a, b \mid x, y)-\left(\frac{1}{\pi_{B}}-1\right) p(a \mid x) \frac{1}{B}-\left(\frac{1}{\pi_{A}}-1\right) \frac{1}{A} p(b \mid y)-\left(\frac{1}{\pi_{A}}-1\right)\left(\frac{1}{\pi_{B}}-1\right) \frac{1}{A B} .
$$

This gives an affine model for $p(a, b \mid x, y)$, as the combination of four local protocols:

1. $P^{\prime}$, with coefficient $\frac{1}{\pi}$;

2. Alice outputs according to $p(a \mid b)$ and Bob outputs uniformly at random, with coefficient $-\left(\frac{1}{\pi_{B}}-1\right)$;

3. Bob outputs according to $p(b \mid y)$ and Alice outputs uniformly at random, with coefficient $-\left(\frac{1}{\pi_{A}}-1\right)$;

4. Both output uniformly at random, with coefficient $-\left(\frac{1}{\pi_{A}}-1\right)\left(\frac{1}{\pi_{B}}-1\right)$.

Then $\tilde{\nu}(\mathbf{p}) \leq \frac{1}{\pi}+\left(\frac{1}{\pi_{B}}-1\right)+\left(\frac{1}{\pi_{A}}-1\right)+\left(\frac{1}{\pi_{A}}-1\right)\left(\frac{1}{\pi_{B}}-1\right)=\frac{2}{\pi}-1 \leq 2^{c+1}-1$.

To get the stronger bound in the binary case, see Appendix $B$

This implies the following lower bounds on classical and quantum communication complexity:

Corollary 14. For any non-signaling distribution $\mathbf{p}$,

1. $R_{0}(\mathbf{p}) \geq \log (\tilde{\nu}(\mathbf{p}))-1$, and $R_{\epsilon}(\mathbf{p}) \geq \log \left(\tilde{\nu}^{\epsilon}(\mathbf{p})\right)-1$.

2. $Q_{0}(\mathbf{p}) \geq \frac{1}{2} \log \left(\tilde{\gamma}_{2}(\mathbf{p})\right)-1$, and $Q_{\epsilon}(\mathbf{p}) \geq \frac{1}{2} \log \left(\tilde{\gamma}_{2}^{\epsilon}(\mathbf{p})\right)-1$.

3. $Q_{0}(C) \geq \log \left(\tilde{\gamma}_{2}(C)\right)$, and $Q_{\epsilon}(C) \geq \log \left(\tilde{\gamma}_{2}^{\epsilon}(C)\right)$. 


\subsection{Factorization norm and related measures}

In the special case of distributions over binary variables with uniform marginals, the quantities $\tilde{\nu}$ and $\tilde{\gamma}_{2}$ become equivalent to the original quantities defined in [LMSS07, LS08b] (at least for the interesting case of non-local correlations, that is correlations with non-zero communication complexity). When the marginals are uniform we omit them and write $\tilde{\nu}(C)$ and $\tilde{\gamma}_{2}(C)$. The following are reformulations as Minkowski functionals of the definitions appearing in [LMSS07, LS08b].

Definition 8. $\quad \nu(C)=\min \left\{\Lambda>0: \frac{1}{\Lambda} C \in \mathcal{L}_{0}\right\}$,

- $\gamma_{2}(C)=\min \left\{\Lambda>0: \frac{1}{\Lambda} C \in \mathcal{Q}_{0}\right\}$,

- $\nu^{\alpha}(C)=\min \left\{\nu\left(C^{\prime}\right): 1 \leq C(x, y) C^{\prime}(x, y) \leq \alpha, \forall x, y \in \mathcal{X} \times \mathcal{Y}\right\}$,

- $\gamma_{2}^{\alpha}(C)=\min \left\{\gamma_{2}\left(C^{\prime}\right): 1 \leq C(x, y) C^{\prime}(x, y) \leq \alpha, \forall x, y \in \mathcal{X} \times \mathcal{Y}\right\}$.

Lemma 15. For any correlation $C: \mathcal{X} \times \mathcal{Y} \rightarrow[-1,1]$,

1. $\tilde{\nu}(C)=1$ iff $\nu(C) \leq 1$, and $\tilde{\gamma}_{2}(C)=1$ iff $\gamma_{2}(C) \leq 1$,

2. $\tilde{\nu}(C)>1 \Longrightarrow \nu(C)=\tilde{\nu}(C)$,

3. $\tilde{\gamma}_{2}(C)>1 \Longrightarrow \gamma_{2}(C)=\tilde{\gamma}_{2}(C)$.

Proof. The first item follows by definition of $\nu$ and $\gamma_{2}$. For the next items, we give the proof for $\nu$, and the proof for $\gamma_{2}$ is similar. The key to the proof is that if $C \in \mathcal{L}_{0}$, then $-C \in \mathcal{L}_{0}$ (it suffices for one of the players to flip his output).

$[\tilde{\nu}(C) \leq \nu(C)]$ If $\tilde{\nu}(C)>1$, then $\Lambda=\nu(C)>1$. Let $C^{+}=\frac{C}{\Lambda}$ and $C^{-}=-\frac{C}{\Lambda}$. By definition of $\nu(C)$, both $C^{+}$ and $C^{-}$are in $\mathcal{L}_{0}$. Furthermore, let $q_{+}=\frac{1+\Lambda}{2} \geq 0$ and $q_{-}=\frac{1-\Lambda}{2} \leq 0$. Since $C=q_{+} C^{+}+q_{-} C^{-}$, this determines an affine model for $C$ with $\left|q_{+}\right|+\left|q_{-}\right|=\Lambda$.

$[\tilde{\nu}(C) \geq \nu(C)]$ Let $\Lambda=\tilde{\nu}(C)$. By definition of $\tilde{\nu}(C)$, there exists $C_{i}$ and $q_{i}$ such that $C=\sum_{i} q_{i} C_{i}$ and $\Lambda=\sum_{i}\left|q_{i}\right|$. Let $\tilde{C}_{i}=\operatorname{sgn}\left(q_{i}\right) C_{i}$ and $p_{i}=\frac{\left|q_{i}\right|}{\Lambda}$. Then, $\frac{C}{\Lambda}=\sum_{i} p_{i} \tilde{C}_{i}$ and therefore $\frac{1}{\Lambda} C \in \mathcal{L}_{0}$ since $\tilde{C}_{i} \in \mathcal{L}_{0}$.

In the special case of sign matrices (corresponding to Boolean functions, as shown above), we also have the following correspondence between $\tilde{\nu}^{\epsilon}, \tilde{\gamma}_{2}^{\epsilon}$, and $\nu^{\alpha}, \gamma_{2}^{\alpha}$.

Lemma 16. Let $0 \leq \epsilon<1 / 2$ and $\alpha=\frac{1}{1-2 \epsilon}$. For any sign matrix $C: \mathcal{X} \times \mathcal{Y} \rightarrow\{-1,1\}$,

1. $\tilde{\nu}^{\epsilon}(C)>1 \Longrightarrow \nu^{\alpha}(C)=\frac{\tilde{\nu}^{\epsilon}(C)}{1-2 \epsilon}$,

2. $\tilde{\gamma}_{2}^{\epsilon}(C)>1 \Longrightarrow \gamma_{2}^{\alpha}(C)=\frac{\tilde{\gamma}_{2}^{\epsilon}(C)}{1-2 \epsilon}$.

Proof. We give the proof for $\nu^{\alpha}$, the proof for $\gamma_{2}^{\alpha}$ is similar.

$\left[\nu^{\alpha}(C) \leq \frac{\tilde{\nu}^{\epsilon}(C)}{1-2 \epsilon}\right]$ By definition of $\tilde{\nu}^{\epsilon}(C)$, there exists a correlation matrix $C^{\prime}$ such that $\tilde{\nu}\left(C^{\prime}\right)=\tilde{\nu}^{\epsilon}(C)$ and $\left|C(x, y)-C^{\prime}(x, y)\right| \leq 2 \epsilon$ for all $x, y \in \mathcal{X} \times \mathcal{Y}$. Since $C$ is a sign matrix, and $C^{\prime}$ is a correlation matrix, $\operatorname{sgn}\left(C^{\prime}(x, y)\right)=$ $C(x, y)$ and $1-2 \epsilon \leq\left|C^{\prime}(x, y)\right| \leq 1$. Hence $1 \leq C(x, y) \frac{C^{\prime}(x, y)}{1-2 \epsilon} \leq \frac{1}{1-2 \epsilon}=\alpha$ This implies that $\nu^{\alpha}(C) \leq \nu\left(\frac{C^{\prime}}{1-2 \epsilon}\right)=$ $\frac{\nu\left(C^{\prime}\right)}{1-2 \epsilon}=\frac{\tilde{\nu}\left(C^{\prime}\right)}{1-2 \epsilon}$, where we used the fact that $\nu\left(C^{\prime}\right)=\tilde{\nu}\left(C^{\prime}\right)$ since $\tilde{\nu}\left(C^{\prime}\right)>1$.

$\left[\nu^{\alpha}(C) \geq \frac{\tilde{\nu}^{\epsilon}(C)}{1-2 \epsilon}\right]$ By definition of $\nu^{\alpha}(C)$, there exists a (not necessarily correlation) matrix $C^{\prime}$ such that $\nu\left(C^{\prime}\right)=$ $\nu^{\alpha}(C)$ and $1 \leq C(x, y) C^{\prime}(x, y) \leq \alpha$ for all $x, y$. Since $C$ is a sign matrix, this implies $\operatorname{sgn}\left(C^{\prime}(x, y)\right)=C(x, y)$ and $1-2 \epsilon \leq\left|\frac{C^{\prime}(x, y)}{\alpha}\right| \leq 1$. Therefore, $\left|C(x, y)-\frac{C^{\prime}(x, y)}{\alpha}\right| \leq 2 \epsilon$ for all $x, y$. This implies that $\tilde{\nu}^{\epsilon}(C) \leq \tilde{\nu}\left(\frac{C^{\prime}}{\alpha}\right)=\nu\left(\frac{C^{\prime}}{\alpha}\right)=$ $(1-2 \epsilon) \nu\left(C^{\prime}\right)$, where we have used the fact that $\tilde{\nu}\left(\frac{C^{\prime}}{\alpha}\right)=\nu\left(\frac{C^{\prime}}{\alpha}\right)$ since $\tilde{\nu}\left(\frac{C^{\prime}}{\alpha}\right) \geq \tilde{\nu}^{\epsilon}(C)>1$.

Just as the special case $\nu(C), \tilde{\nu}(\mathbf{p})$ may be expressed as a linear program. However, while $\gamma_{2}(C)$ could be expressed as a semidefinite program, this may not be true in general for $\tilde{\gamma}_{2}(\mathbf{p})$. Nevertheless, using the hierarchy $\left\{\mathcal{Q}^{n}: n \in \mathbb{N}_{0}\right\}$ introduced in [NPA08], it admits SDP relaxations $\left\{\tilde{\gamma}_{2}^{n}(\mathbf{p}): n \in \mathbb{N}_{0}\right\}$.

Definition 9. $\tilde{\gamma}_{2}^{n}(\mathbf{p})=\min \left\{\sum_{i}\left|q_{i}\right|: \exists \mathbf{p}_{i} \in \mathcal{Q}^{n}, q_{i} \in \mathbb{R}, \mathbf{p}=\sum_{i} q_{i} \mathbf{p}_{i}\right\}$. 
The fact that $\mathcal{Q}^{n} \subseteq \mathcal{Q}^{n-1}$ implies $\tilde{\gamma}_{2}^{n}(\mathbf{p}) \geq \tilde{\gamma}_{2}^{n-1}(\mathbf{p})$, and by continuity of the minimization function, $\tilde{\gamma}_{2}^{n}(\mathbf{p}) \rightarrow$ $\tilde{\gamma}_{2}(\mathbf{p})$ for $n \rightarrow \infty$.

Lemmas 15 and 16 establish that Corollary 14 is a generalization of Linial and Shraibman's factorization norm lower bound technique. Note that unlike Linial and Shraibman, we do not obtain the stronger statement that $R_{\epsilon}(C) \geq$ $2 \log \left(\gamma_{2}^{\epsilon}(C)\right)$, only that $R_{\epsilon}(C) \geq \log \left(\gamma_{2}^{\epsilon}(C)\right)$. This may be due to the fact that the result holds in the much more general setting of non-signaling distributions with arbitrary outcomes and marginals.

Because of Proposition 9 , we know that $\nu(C) \leq K_{G} \gamma_{2}(C)$ for correlations. Such a bound is not known for $\tilde{\nu}$ and $\tilde{\gamma}_{2}$, so there may still be an exponential gap between these two quantities.

Note also that although $\gamma_{2}$ and $\nu$ are matrix norms, this fails to be the case for $\tilde{\gamma}_{2}$ and $\tilde{\nu}$, even in the case of correlations. Nevertheless, it is still possible to formulate dual quantities, which turn out to have sufficient structure, as we show in the next section.

\section{Duality, Bell inequalities, and XOR games}

In their primal formulation, the $\tilde{\gamma}_{2}$ and $\tilde{\nu}$ methods are difficult to apply since they are formulated as a minimization problem. Transposing to the dual space not only turns the method into a maximization problem; it also has a very natural, well-understood interpretation since it coincides with maximal violations of Bell and Tsirelson inequalities. This is particularly relevant to physics, since it formalizes in very precise terms the intuition that distributions with large Bell inequality violations should require more communication to simulate.

Recall that for any norm $\|\cdot\|$ on a vector space $V$, the dual norm is $\|B\|^{*}=\max _{v \in V:\|v\| \leq 1} B(v)$, where $B$ is a linear functional on $V$.

\subsection{Bell and Tsirelson inequalities}

Bell inequalities were first introduced by Bell [Bel64], as bounds on the correlations that could be achieved by any local physical theory. He showed that quantum correlations could violate these inequalities and therefore exhibited non-locality. Tsirelson later proved that quantum correlations should also respect some bound (known as the Tsirelson bound), giving a first example of a "Tsirelson-like" inequality for quantum distributions [Tsi80].

Since the set of non-signaling distributions $\mathcal{C}$ lies in an affine space aff $(\mathcal{C})$, we may consider the isomorphic dual space of linear functionals over this space. The dual quantity $\tilde{\nu}^{*}$ (technically not a dual norm since $\tilde{\nu}$ itself is not a norm in the general case) is the maximum value of a linear functional in the dual space on local distributions, and $\tilde{\gamma}_{2}^{*}$ is the maximum value of a linear functional on quantum distributions. These are exactly what is captured by the Bell and Tsirelson inequalities.

Definition 10 (Bell and Tsirelson inequalities). Let $B: \operatorname{aff}(\mathcal{C}) \mapsto \mathbb{R}$ be a linear functional on the (affine hull of the) set of non-signaling distributions, $B(\mathbf{p})=\sum_{a, b, x, y} B_{a b x y} p(a, b \mid x, y)$. Define $\tilde{\nu}^{*}(B)=\max _{\mathbf{p} \in \mathcal{L}} B(\mathbf{p})$ and $\tilde{\gamma}_{2}^{*}(B)=$ $\max _{\mathbf{p} \in \mathcal{Q}} B(\mathbf{p})$. A Bell inequality is a linear inequality satisfied by any local distribution, $B(\mathbf{p}) \leq \tilde{\nu}^{*}(B)(\forall \mathbf{p} \in \mathcal{L})$, and a Tsirelson inequality is a linear inequality satisfied by any quantum distribution, $B(\mathbf{p}) \leq \tilde{\gamma}_{2}^{*}(B)(\forall \mathbf{p} \in \mathcal{Q})$.

By linearity (Proposition 1) Bell inequalities are often expressed as linear functionals over the correlations in the case of binary outputs and uniform marginals.

Finally, $\tilde{\gamma}_{2}$ and $\tilde{\nu}$ amount to finding a maximum violation of a (normalized) Bell or Tsirelson inequality.

Theorem 17. For any distribution $\mathbf{p} \in \mathcal{C}$,

$$
\begin{aligned}
& \text { 1. } \tilde{\nu}(\mathbf{p})=\max \left\{B(\mathbf{p}): \forall \mathbf{p}^{\prime} \in \mathcal{L},\left|B\left(\mathbf{p}^{\prime}\right)\right| \leq 1\right\} \text {, and } \\
& \text { 2. } \tilde{\gamma}_{2}(\mathbf{p})=\max \left\{B(\mathbf{p}): \forall \mathbf{p}^{\prime} \in \mathcal{Q},\left|B\left(\mathbf{p}^{\prime}\right)\right| \leq 1\right\} \text {, }
\end{aligned}
$$

where the maximization is over linear functionals $B: \operatorname{aff}(\mathcal{C}) \mapsto \mathbb{R}$.

Proof. 1. This follows by LP duality from the definition of $\tilde{\nu}$. 
2. We use the SDP relaxation $\tilde{\gamma}_{2}^{n}(\mathbf{p})$, which may be expressed as

$$
\tilde{\gamma}_{2}^{n}(\mathbf{p})=\min \left\{q_{+}+q_{-}: \exists \mathbf{p}_{+}, \mathbf{p}_{-} \in \mathcal{Q}^{n}, q_{+}, q_{-} \geq 0, \mathbf{p}=q_{+} \mathbf{p}_{+}-q_{-} \mathbf{p}_{-}\right\}
$$

and define

$$
\beta^{n}(\mathbf{p})=\max \left\{B(\mathbf{p}): \forall \mathbf{p}^{\prime} \in \mathcal{Q}^{n},\left|B\left(\mathbf{p}^{\prime}\right)\right| \leq 1\right\} .
$$

We now show that $\beta^{n}(\mathbf{p})=\tilde{\gamma}_{2}^{n}(\mathbf{p})$, which proves our statement by taking the limit $n \rightarrow \infty$.

$\left[\beta^{n}(\mathbf{p}) \leq \tilde{\gamma}_{2}^{n}(\mathbf{p})\right]$ Let $\tilde{\gamma}_{2}^{n}(\mathbf{p})=q_{+}+q_{-}$, where $q_{+}, q_{-} \geq 0$ and $\mathbf{p}=q_{+} \mathbf{p}_{+}-q_{-} \mathbf{p}_{-}$for some $\mathbf{p}_{+}, \mathbf{p}_{-} \in \mathcal{Q}^{n}$. Similarly, let $\beta^{n}(\mathbf{p})=B(\mathbf{p})$, where $\left|B\left(\mathbf{p}^{\prime}\right)\right| \leq 1$ for all $\mathbf{p}^{\prime} \in \mathcal{Q}^{n}$. It then follows that

$$
B(\mathbf{p})=q_{+} B\left(\mathbf{p}_{+}\right)-q_{-} B\left(\mathbf{p}_{-}\right) \leq q_{+}\left|B\left(\mathbf{p}_{+}\right)\right|+q_{-}\left|B\left(\mathbf{p}_{-}\right)\right| \leq q_{+}+q_{-} .
$$

$\left[\beta^{n}(\mathbf{p}) \geq \tilde{\gamma}_{2}^{n}(\mathbf{p})\right]$ In order to use SDP duality, we first express $\tilde{\gamma}_{2}^{n}(\mathbf{p})$ in standard SDP form. Using the definition of $\mathcal{Q}^{n}$,

$$
\begin{aligned}
\tilde{\gamma}_{2}^{n}(\mathbf{p})=\min & \Gamma_{\mathbb{1}, \mathbb{1}}^{+}+\Gamma_{\mathbb{1}, \mathbb{1}}^{-} \\
\text {subject to } \quad & \Gamma^{+}, \Gamma^{-} \succcurlyeq 0, \\
& \Gamma_{E_{a}(x), E_{b}(y)}^{+}-\Gamma_{E_{a}(x), E_{b}(y)}^{-}=p(a, b \mid x, y), \\
& \operatorname{tr}\left(F_{k}^{\dagger} \Gamma^{+}\right)=\operatorname{tr}\left(F_{k}^{\dagger} \Gamma^{-}\right)=0 \quad \forall k \in[m(n)] .
\end{aligned}
$$

The dual SDP then reads

$$
\begin{aligned}
& \delta^{n}(\mathbf{p})=\max \sum_{a, b, x, y} B_{a b x y} p(a, b \mid x, y) \\
& \text { subject to } \sum_{a, b, x, y} B_{a b x y} \Gamma_{E_{a}(x), E_{b}(y)} \geq-\left[\Gamma_{\mathbb{1}, \mathbb{1}}+\sum_{k \in[m(n)]} B_{k}^{-} \operatorname{tr}\left(F_{k}^{\dagger} \Gamma\right)\right] \quad \forall \Gamma \succcurlyeq 0 \text {, } \\
& \sum_{a, b, x, y} B_{a b x y} \Gamma_{E_{a}(x), E_{b}(y)} \leq \Gamma_{\mathbb{1}, \mathbb{1}}+\sum_{k \in[m(n)]} B_{k}^{+} \operatorname{tr}\left(F_{k}^{\dagger} \Gamma\right) \quad \forall \Gamma \succcurlyeq 0 .
\end{aligned}
$$

It may be shown that the dual is strictly feasible, so that strong duality holds and $\delta^{n}(\mathbf{p})=\tilde{\gamma}_{2}^{n}(\mathbf{p})(\mathrm{see}[\mathrm{VB} 96])$. Together with the definition of $\mathcal{Q}^{n}$, this shows that a feasible solution for $\delta^{n}(\mathbf{p})$ implies a feasible solution for $\beta^{n}(\mathbf{p})$, so that $\beta^{n}(\mathbf{p}) \geq \delta^{n}(\mathbf{p})$.

\subsection{XOR games}

In this section, we consider distributions over binary variables with uniform marginals, $\mathbf{p}=(C, 0,0)$, and furthermore restrict to the case of sign matrices $C \in\{ \pm 1\}^{\mathcal{X} \times \mathcal{Y}}$. As we have seen before, this corresponds to the standard framework of communication complexity of Boolean functions, and we have $\tilde{\nu}(C, 0,0)=\nu(C)$. We show a close relation between $\nu(C)$, XOR games and Bell inequalities.

In an XOR game, Alice is given some input $x$ and Bob is given an input $y$, and they should output $a= \pm 1$ and $b= \pm 1$. They win if $a \cdot b$ equals some \pm 1 function $G(x, y)$. Since they are not allowed to communicate, their strategy may be represented as a local correlation matrix $S \in \mathcal{L}_{0}$. We consider the distributional version of this game, where $\mu$ is a distribution on the inputs. Define the winning bias given some strategy $S$ with respect to $\mu$ to be $\epsilon_{\mu}(G \| S)=\sum_{x, y} \mu(x, y) G(x, y) S(x, y)$, and $\epsilon_{\mu}(G)=\max _{S \in \mathcal{L}_{0}} \epsilon_{\mu}(G \| S)$ be the maximum winning bias of any local (classical) strategy. (For convenience, we consider the bias instead of game value $\omega_{\mu}(G)=\left(1+\epsilon_{\mu}(G)\right) / 2$.) Define $\epsilon_{\mu}^{\mathrm{ent}}(G)$ similarly for quantum strategies. When the input distribution is not fixed, we define the game biases as $\epsilon(G)=\min _{\mu} \epsilon_{\mu}(G)$ and $\epsilon^{\mathrm{ent}}(G)=\min _{\mu} \epsilon_{\mu}^{\mathrm{ent}}(G)$.

Lemma 18. There is a bijection between XOR games $(G, \mu)$ and normalized correlation Bell inequalities. 
Proof. An XOR game $(G, \mu)$ determines a linear functional $G \circ \mu(C)=\epsilon_{\mu}(G \| C)$ on the set of correlation matrices, where $\circ$ is the Hadamard (entrywise) product. By Definition 10, $\nu^{*}(G \circ \mu)=\epsilon_{\mu}(G)$, and $\epsilon_{\mu}(G \| C) \leq \epsilon_{\mu}(G)$ is a Bell inequality satisfied by any local correlation matrix $C$. Similarly, when the players are allowed to use entanglement, we get a Tsirelson inequality on quantum correlations, $\epsilon_{\mu}^{\mathrm{ent}}(G \| C) \leq \epsilon_{\mu}^{\mathrm{ent}}(G)$ (the quantum bias is also equivalent to a dual norm $\epsilon_{\mu}^{\mathrm{ent}}(G)=\gamma_{2}^{*}(G \circ \mu)$ ).

Conversely, consider a general linear functional $B(C)=\sum_{x, y} B_{x y} C(x, y)$ on aff $\left(\mathcal{C}_{0}\right)$, defining a correlation Bell inequality $B(C) \leq \nu^{*}(B) \forall C \in \mathcal{L}_{0}$. Dividing this Bell inequality by $N=\sum_{x, y}\left|B_{x y}\right|$, we see that it determines an XOR game specified by a sign matrix $G(x, y)=\operatorname{sgn}\left(B_{x y}\right)$ and an input distribution $\mu_{x y}=\frac{\left|B_{x y}\right|}{N}$, and having a game bias $\epsilon_{\mu}(G)=\frac{\nu^{*}(B)}{N}$.

By Theorem 17 and the previous bijection (see also Lee et al. [LSŠ08]):

Corollary 19. 1. $\nu(C)=\max _{\mu, G} \frac{\epsilon_{\mu}(G \| C)}{\epsilon_{\mu}(G)}$ where the maximum is over XOR games $(G, \mu)$.

2. $\nu(C) \geq \frac{1}{\epsilon(C)}$.

The second part follows by letting $G=C$. Even though playing correlations $C$ for a game $G=C$ allows us to win with probability one, there are cases where some other game $G \neq C$ yields a larger ratio. In these cases, we have $\nu(C)>\frac{1}{\epsilon(C)}$ so that $\nu$ gives a stronger lower bound for communication complexity than the game value (which has been shown to be equivalent to the discrepancy method [LSŠ08]).

We can characterize when the inequality is tight. Let $\epsilon^{=}(C)=\max _{S \in \mathcal{L}_{0}}\{\beta: \forall x, y, C(x, y) S(x, y)=\beta\}$, that is, we only consider strategies that wins the game with equal bias with respect to all distributions. For the sake of comparison, the game bias may also be expressed as $\left.\epsilon(C)=\max _{S \in \mathcal{L}_{0}}\{\beta: \forall x, y, C(x, y) S(x, y) \geq \beta\}=\max _{S \in \mathcal{L}_{0}} \min _{x, y} C(x, y) S(x, y)\right\}$ [vN2

Lemma 20. $\nu(C)=\frac{1}{\epsilon^{=}(C)}$.

We can also relate the game value to $\nu^{\alpha}(C)$, as it was shown in [LSŠ08] that for $\alpha \rightarrow \infty, \nu^{\infty}(C)$ is exactly the inverse of the game bias $\frac{1}{\epsilon(C)}$. We show that this holds as soon as $\alpha=\frac{1}{1-2 \epsilon}$ is large enough for $C$ to be local up to an error $\epsilon$, completing the picture given in Lemma 16 .

Lemma 21. Let $0 \leq \epsilon<1 / 2$ and $\alpha=\frac{1}{1-2 \epsilon}$. For any sign matrix $C: \mathcal{X} \times \mathcal{Y} \rightarrow\{-1,1\}$,

1. $\tilde{\nu}^{\epsilon}(C)=1 \Longleftrightarrow \epsilon \geq 1-\omega(C) \Longleftrightarrow \alpha \geq \frac{1}{\epsilon(C)} \Longleftrightarrow \nu^{\alpha}(C)=\nu^{\infty}(C)=\frac{1}{\epsilon(C)}$

2. $\tilde{\gamma}_{2}^{\epsilon}(C)=1 \Longleftrightarrow \epsilon \geq 1-\omega^{\mathrm{ent}}(C) \Longleftrightarrow \alpha \geq \frac{1}{\epsilon^{\mathrm{ent}}(C)} \Longleftrightarrow \gamma_{2}^{\alpha}(C)=\gamma_{2}^{\infty}(C)=\frac{1}{\epsilon^{\mathrm{ent}}(C)}$

Proof. By von Neumann's minmax principle [vN28],

$$
\begin{aligned}
\epsilon(C) & =\max _{S \in \mathcal{L}_{0}} \min _{x, y} C(x, y) S(x, y) \\
& =\max _{S \in \mathcal{L}_{0}} \min _{x, y} 1-|C(x, y)-S(x, y)|
\end{aligned}
$$

where we used the fact that $C$ is a sign matrix. This implies that $\tilde{\nu}^{\epsilon}(C)=1 \Leftrightarrow \epsilon \geq \frac{1-\epsilon(C)}{2} \Leftrightarrow \alpha \geq \frac{1}{\epsilon(C)}$.

By Lemma 16, this in turn implies that $\nu^{\alpha}(C)=\frac{\tilde{\nu}^{\epsilon}(C)}{1-2 \epsilon}$ for all $\epsilon<\frac{1-\epsilon(C)}{2}$. By continuity, taking the limit $\epsilon \rightarrow \frac{1-\epsilon(C)}{2}$ yields $\nu^{\alpha}(C)=\frac{1}{\epsilon(C)}$ for $\alpha=\frac{1}{\epsilon(C)}$. From [LSŠ08], $\nu^{\infty}(C)=\frac{1}{\epsilon(C)}$, and the lemma follows by the monotonicity of $\nu^{\alpha}(C)$ as a function of $\alpha$.

\section{Comparing $\tilde{\gamma}_{2}$ and $\tilde{\nu}$}

It is known that because of Grothendieck's inequality, $\gamma_{2}$ and $\nu$ differ by at most a constant. We show in this section that this also extends to non-signaling distributions. 
Theorem 22. For any non-signaling distribution $\mathbf{p} \in \mathcal{C}$, with inputs in $\mathcal{X} \times \mathcal{Y}$ and outcomes in $\mathcal{A} \times \mathcal{B}$ with $A=$ $|\mathcal{A}|, B=|\mathcal{B}|$,

1. $\tilde{\nu}(\mathbf{p}) \leq\left(2 K_{G}+1\right) \tilde{\gamma}_{2}(\mathbf{p})$ when $A=B=2$,

2. $\tilde{\nu}(\mathbf{p}) \leq\left[2 A B\left(K_{G}+1\right)-1\right] \tilde{\gamma}_{2}(\mathbf{p})$ for any $A, B$.

The negative consequence of this is that one cannot hope to prove separations between classical and quantum communication using this method, except in the case where the number of outcomes is large. For binary outcomes at least, this says that arguments based on analysing the distance to the quantum set only, without taking into account the particular structure of the distribution, will not suffice to prove large separations; and other techniques, such as information theoretic arguments, may be necessary.

For example, Brassard et al. [BCT99] give a (promise) distribution based on the Deutsch-Jozsa problem, which can be obtained exactly with entanglement and no communication, but which requires linear communication to simulate exactly. The lower bound is proven using a corruption bound [BCW98], which is closely related to the information theoretic subdistribution bound [JKN08]. For this problem, $\mathcal{X}=\mathcal{Y}=\{0,1\}^{n}$ and $\mathcal{A}=\mathcal{B}=[n]$, therefore our method can only prove a lower bound logarithmic in $n$.

On the positive side, this is very interesting for quantum information, since (by Theorem 17), it tells us that the set of quantum distributions cannot be much larger than the local polytope, for any number of inputs and outcomes. For binary correlations, this follows from the theorems of Tsirelson (Theorem 7) and Grothendieck (Proposition 9), but no extensions are known for these results in the more general setting.

The proof will use two rather straightforward lemmas.

Lemma 23. If $\mathbf{p}=\sum_{i \in[I]} q_{i} \mathbf{p}_{i}$, where $\mathbf{p}_{i} \in \mathcal{C}$ and $q_{i} \in \mathbb{R}$ for all $i \in[I]$, then $\tilde{\nu}(\mathbf{p}) \leq \sum_{i \in[I]}\left|q_{i}\right| \tilde{\nu}\left(\mathbf{p}_{i}\right)$.

Proof. By definition, for each $\mathbf{p}_{i}$, there exists $\mathbf{p}_{i}^{+}, \mathbf{p}_{i}^{-} \in \mathcal{L}$ and $q_{i}^{+}, q_{i}^{-} \geq 0$ such that $\mathbf{p}_{i}=q_{i}^{+} \mathbf{p}_{i}^{+}-q_{i}^{-} \mathbf{p}_{i}^{-}$, and $q_{i}^{+}+q_{i}^{-}=\tilde{\nu}\left(\mathbf{p}_{i}\right)$. Therefore, $\mathbf{p}=\sum_{i \in[I]} q_{i}\left(q_{i}^{+} \mathbf{p}_{i}^{+}-q_{i}^{-} \mathbf{p}_{i}^{-}\right)$and $\sum_{i \in[I]}\left(\left|q_{i} q_{i}^{+}\right|+\left|q_{i} q_{i}^{-}\right|\right)=\sum_{i}\left|q_{i}\right|\left(q_{i}^{+}+q_{i}^{-}\right)=$ $\sum_{i}\left|q_{i}\right| \tilde{\nu}\left(\mathbf{p}_{i}\right)$.

Lemma 24. Let $\mathbf{p}, \mathbf{p}^{\prime} \in \mathcal{C}$ be non-signaling distributions with inputs in $\mathcal{X} \times \mathcal{Y}$ for both distributions, outcomes in $\mathcal{A} \times \mathcal{B}$ for $\mathbf{p}$, and outcomes in $\mathcal{A}^{\prime} \times \mathcal{B}^{\prime}$ for $\mathbf{p}^{\prime}$, such that $\mathcal{A} \subseteq \mathcal{A}^{\prime}$ and $\mathcal{B} \subseteq \mathcal{B}^{\prime}$. If, for any $(a, b) \in \mathcal{A} \times \mathcal{B}$ $p^{\prime}(a, b \mid x, y)=p(a, b \mid x, y)$, then $\tilde{\nu}\left(\mathbf{p}^{\prime}\right)=\tilde{\nu}(\mathbf{p})$.

Proof. Let $\mathcal{E}=\left(\mathcal{A}^{\prime} \times \mathcal{B}^{\prime}\right) \backslash(\mathcal{A} \times \mathcal{B})$. First, note that since $p^{\prime}(a, b \mid x, y)=p(a, b \mid x, y)$ for any $(a, b) \in \mathcal{A} \times \mathcal{B}$, we have, by normalization of $\mathbf{p}, p^{\prime}(a, b \mid x, y)=0$ for any $(a, b) \in \mathcal{E}$.

$\left[\tilde{\nu}\left(\mathbf{p}^{\prime}\right) \leq \tilde{\nu}(\mathbf{p})\right]$ Let $\mathbf{p}=q_{+} \mathbf{p}^{+}-q_{-} \mathbf{p}^{-}$be an affine model for $\mathbf{p}$. Obviously, this implies an affine model for $\mathbf{p}^{\prime}$ by extending the local distributions $\mathbf{p}^{+}, \mathbf{p}^{-}$from $\mathcal{A} \times \mathcal{B}$ to $\mathcal{A}^{\prime} \times \mathcal{B}^{\prime}$, by setting $p^{+}(a, b \mid x, y)=p^{-}(a, b \mid x, y)=0$ for any $(a, b) \in \mathcal{E}$, so $\tilde{\nu}\left(\mathbf{p}^{\prime}\right) \leq \tilde{\nu}(\mathbf{p})$.

$\left[\tilde{\nu}\left(\mathbf{p}^{\prime}\right) \geq \tilde{\nu}(\mathbf{p})\right]$ Let $\mathbf{p}^{\prime}=q_{+} \mathbf{p}^{\prime+}-q_{-} \mathbf{p}^{\prime-}$ be an affine model for $\mathbf{p}^{\prime}$. We may not immediately derive an affine model for $\mathbf{p}$ since it could be the case that $p^{\prime+}(a, b \mid x, y)$ or $p^{-}(a, b \mid x, y)$ is non zero for some $(a, b) \in \mathcal{E}$. However, we have $q_{+} p^{\prime+}(a, b \mid x, y)-q_{-} p^{-}(a, b \mid x, y)=p^{\prime}(a, b \mid x, y)=0$ for any $(a, b) \in \mathcal{E}$, so we may define an affine model $\mathbf{p}=q_{+} \mathbf{p}^{+}-q_{-} \mathbf{p}^{-}$, where $\mathbf{p}^{+}$and $\mathbf{p}^{-}$are distributions on $\mathcal{A} \times \mathcal{B}$ such that

$$
p^{+}(a, b \mid x, y)=p^{+}(a, b \mid x, y)+\frac{1}{A} \sum_{a^{\prime} \notin \mathcal{A}} p^{\prime+}\left(a^{\prime}, b \mid x, y\right)+\frac{1}{B} \sum_{b^{\prime} \notin \mathcal{B}} p^{\prime+}\left(a, b^{\prime} \mid x, y\right)+\frac{1}{A B} \sum_{a^{\prime} \notin \mathcal{A}, b^{\prime} \notin \mathcal{B}} p^{\prime+}\left(a^{\prime}, b^{\prime} \mid x, y\right),
$$

and similarly for $\mathbf{p}^{-}$. These are local since it suffices for Alice and Bob to use the local protocol for $\mathbf{p}^{\prime+}$ or $\mathbf{p}^{\prime-}$ and for Alice to replace any output $a \notin \mathcal{A}$ by a uniformly random output $a^{\prime} \in \mathcal{A}$ (similarly for Bob). Therefore, we also have $\tilde{\nu}\left(\mathbf{p}^{\prime}\right) \geq \tilde{\nu}(\mathbf{p})$.

Before proving Theorem 22, we first consider the special case of quantum distributions, such that $\tilde{\gamma}_{2}(\mathbf{p})=1$. As we shall see in Section 6 , this special case implies the constant upper bound of Shi and Zhu on approximating any quantum distribution [SZ08], which they prove using diamond norms. This also immediately gives an upper bound on maximum Bell inequality violations for quantum distributions, by Theorem 17, which may be of independent interest in quantum information theory. 
Proposition 25. For any quantum distribution $\mathbf{p} \in \mathcal{Q}$, with inputs in $\mathcal{X} \times \mathcal{Y}$ and outcomes in $\mathcal{A} \times \mathcal{B}$ with $A=$ $|\mathcal{A}|, B=|\mathcal{B}|$,

1. $\tilde{\nu}(\mathbf{p}) \leq 2 K_{G}+1$ when $A=B=2$,

2. $\tilde{\nu}(\mathbf{p}) \leq 2 A B\left(K_{G}+1\right)-1$ for any $A, B$.

Proof. 1. Since $A=B=2$, we may write the distribution as correlations and marginals, $\mathbf{p}=\left(C, M_{A}, M_{B}\right)$. Since $\left(C, M_{A}, M_{B}\right) \in \mathcal{Q}$, we also have $(C, 0,0) \in \mathcal{Q}$, and by Tsirelson's theorem, $\left(C / K_{G}, 0,0\right) \in \mathcal{L}$. Moreover, it is immediate that $\left(M_{A} M_{B}, M_{A}, M_{B}\right),\left(M_{A} M_{B}, 0,0\right)$ and $(0,0,0)$ are local distributions as well, so that we have the following affine model for $\left(C, M_{A}, M_{B}\right)$

$$
\left(C, M_{A}, M_{B}\right)=K_{G}\left(C / K_{G}, 0,0\right)+\left(M_{A} M_{B}, M_{A}, M_{B}\right)-\left(M_{A} M_{B}, 0,0\right)-\left(K_{G}-1\right)(0,0,0) .
$$

This implies that $\tilde{\nu}\left(C, M_{A}, M_{B}\right) \leq 2 K_{G}+1$.

2. For the general case, we will reduce to the binary case. Let us introduce an additional output $\varnothing$, and set $\mathcal{A}^{\prime}=\mathcal{A} \cup\{\varnothing\}$ and $\mathcal{B}^{\prime}=\mathcal{B} \cup\{\varnothing\}$. We first extend the distribution $\mathbf{p}$ to a distribution $\mathbf{p}^{\prime}$ on $\mathcal{A}^{\prime} \times \mathcal{B}^{\prime}$ by setting $p^{\prime}(a, b \mid x, y)=p(a, b \mid x, y)$ for any $(a, b) \in \mathcal{A} \times \mathcal{B}$, and $p^{\prime}(a, b \mid x, y)=0$ otherwise. By Lemma 24 we have $\tilde{\nu}(\mathbf{p})=\tilde{\nu}\left(\mathbf{p}^{\prime}\right)$.

For each $(\alpha, \beta) \in \mathcal{A} \times \mathcal{B}$, we also define a probability distribution $\mathbf{p}_{\alpha \beta}$ on $\mathcal{A}^{\prime} \times \mathcal{B}^{\prime}$, such that

$$
p_{\alpha \beta}(a, b \mid x, y)= \begin{cases}p(\alpha, \beta \mid x, y) & \text { if }(a, b)=(\alpha, \beta), \\ 1-p(\alpha, \beta \mid x, y) & \text { if }(a, b)=(\varnothing, \varnothing), \\ 0 & \text { otherwise }\end{cases}
$$

Let $\mathcal{A}_{\alpha}=\{\alpha, \varnothing\}$ and $\mathcal{B}_{\beta}=\{\beta, \varnothing\}$. Since $p_{\alpha \beta}(a, b \mid x, y)=0$ when $(a, b) \notin \mathcal{A}_{\alpha} \times \mathcal{B}_{\beta}$, we may define distributions $\mathbf{p}_{\alpha \beta}^{\prime}$ on $\mathcal{A}_{\alpha} \times \mathcal{B}_{\beta}$ such that $p_{\alpha \beta}^{\prime}(a, b \mid x, y)=p_{\alpha \beta}(a, b \mid x, y)$ for all $(a, b) \in \mathcal{A}_{\alpha} \times \mathcal{B}_{\beta}$. By Lemma24, these are such that $\tilde{\nu}\left(\mathbf{p}_{\alpha \beta}^{\prime}\right)=\tilde{\nu}\left(\mathbf{p}_{\alpha \beta}\right)$, and since these are binary distributions, $\tilde{\nu}\left(\mathbf{p}_{\alpha \beta}^{\prime}\right) \leq 2 K_{G}+1$. Moreover, let us define a distribution $\mathbf{p}_{\varnothing}$ on $\mathcal{A}^{\prime} \times \mathcal{B}^{\prime}$ such that $p_{\varnothing}(a, b \mid x, y)=1$ if $(a, b)=(\varnothing, \varnothing)$, and $p_{\varnothing}(a, b \mid x, y)=0$ otherwise. This is a deterministic distribution, so $\mathbf{p}_{\varnothing} \in \mathcal{L}$ and $\tilde{\nu}\left(\mathbf{p}_{\varnothing}\right)=1$. We may now build the following affine model for $\mathbf{p}^{\prime}$

$$
\mathbf{p}^{\prime}=\sum_{(\alpha, \beta) \in \mathcal{A} \times \mathcal{B}} \mathbf{p}_{\alpha \beta}-(A B-1) \mathbf{p}_{\varnothing}
$$

From Lemma23, we conclude that $\tilde{\nu}\left(\mathbf{p}^{\prime}\right) \leq A B\left(2 K_{G}+1\right)+(A B-1)=2 A B\left(K_{G}+1\right)-1$

The proof of Theorem 22 immediately follows.

Proof of Theorem 22 By definition of $\tilde{\gamma}_{2}(\mathbf{p})$, there exists $\mathbf{p}^{+}, \mathbf{p}^{-} \in \mathcal{Q}$ and $q_{+}, q_{-} \geq 0$ such that $\mathbf{p}=q_{+} \mathbf{p}^{+}-q_{-} \mathbf{p}^{-}$ and $q_{+}+q_{-}=\tilde{\gamma}_{2}(\mathbf{p})$. From Lemma 23, $\tilde{\nu}(\mathbf{p}) \leq q_{+} \tilde{\nu}\left(\mathbf{p}^{+}\right)+q_{-} \tilde{\nu}\left(\mathbf{p}^{-}\right)$, and Proposition 25 immediately concludes the proof.

\section{Upper bounds for non-signaling distributions}

We have seen that if a distribution can be simulated using $t$ bits of communication, then it may be represented by an affine model with coefficients exponential in $t$ (Theorem 13). In this section, we consider the converse: how much communication is required to simulate a distribution, given an affine model? This approach allows us to show that any (shared randomness or entanglement-assisted) communication protocol can be simulated with simultaneous messages, with an exponential cost to the simulation, which was previously known only in the case of Boolean functions [Yao03, SZ08, GKd06]. We use the superscript $\|$ to indicate the simultaneous messages model, where Alice and Bob each send a message to the referee, who without knowing the inputs, outputs the value of the function, or more generally, outputs $a, b$ with the correct probability distribution conditioned on the inputs $x, y$. 
Theorem 26. For any distribution $\mathbf{p} \in \mathcal{C}$ with inputs in $\mathcal{X} \times \mathcal{Y}$ with $|\mathcal{X} \times \mathcal{Y}| \leq 2^{n}$, and outcomes in $\mathcal{A} \times \mathcal{B}$ with $A=|\mathcal{A}|, B=|\mathcal{B}|$, and any $\epsilon, \delta<1 / 2$,

$$
\begin{aligned}
& \text { 1. } R_{\epsilon+\delta}^{\|, \mathrm{pub}}(\mathbf{p}) \leq 16\left[\frac{A B \tilde{\nu}^{\epsilon}(p)}{\delta}\right]^{2} \ln \left[\frac{4 A B}{\delta}\right] \log (A B), \\
& \text { 2. } Q_{\epsilon+\delta}^{\|}(\mathbf{p}) \leq O\left((A B)^{5}\left[\frac{\tilde{\nu}^{\epsilon}(p)}{\delta}\right]^{4} \ln \left[\frac{A B}{\delta}\right] \log (n)\right) .
\end{aligned}
$$

The proof relies on Hoeffding's inequality [McD91].

Proposition 27 (Hoeffding's inequality). Let $X$ be a random variable with values in $[a, b]$. Let $X_{t}$ be the t-th of $T$ independent trials of $X$, and $S=\frac{1}{T} \sum_{t=1}^{T} X_{t}$.

Then, $\operatorname{Pr}[S-E(X) \geq \beta] \leq e^{-\frac{2 T \beta^{2}}{(b-a)^{2}}}$, and $\operatorname{Pr}[E(X)-S \geq \beta] \leq e^{-\frac{2 T \beta^{2}}{(b-a)^{2}}}$, for any $\beta \geq 0$.

We will also use the following lemma.

Lemma 28. Let $\mathbf{p}$ be a probability distribution on $\mathcal{V}$ with $V=|\mathcal{V}|$, and $e: \mathbb{R}^{+} \rightarrow \mathbb{R}^{+}$. For each $v \in \mathcal{V}$, let $Q_{v}$ be a random variable such that $\forall \beta \geq 0, \operatorname{Pr}\left[Q_{v} \geq p(v)+\beta\right] \leq e(\beta)$ and $\operatorname{Pr}\left[Q_{v} \leq p(v)-\beta\right] \leq e(\beta)$.

Then, given samples $\left\{Q_{v}: v \in \mathcal{V}\right\}$, and without knowing $\mathbf{p}$, we may simulate a probability distribution $\mathbf{p}^{\prime}$ such that $\delta\left(\mathbf{p}^{\prime}, \mathbf{p}\right) \leq 2 V[\beta+e(\beta)]$.

Proof. In order to use the variables $Q_{v}$ as estimations for $p(v)$, we must first make them positive, and then renormalize them so that they sum up to 1 . Let $R_{v}=\max \left\{0, Q_{v}\right\}$. Then we may easily verify that

$$
\begin{aligned}
& \operatorname{Pr}\left[R_{v} \geq p(v)+\beta\right] \leq e(\beta), \\
& \operatorname{Pr}\left[R_{v} \leq p(v)-\beta\right] \leq e(\beta) .
\end{aligned}
$$

For any subset $\mathcal{E} \subseteq \mathcal{V}$ of size $E=|\mathcal{E}|$, we also define the estimates $R_{\mathcal{E}}=\sum_{v \in \mathcal{E}} R_{v}$ for $p(\mathcal{E})$. By summing,

$$
\begin{aligned}
& \operatorname{Pr}\left[R_{\mathcal{E}} \geq p(\mathcal{E})+E \beta\right] \leq E e(\beta) \\
& \operatorname{Pr}\left[R_{\mathcal{E}} \leq p(\mathcal{E})-E \beta\right] \leq E e(\beta)
\end{aligned}
$$

In order to renormalize the estimated probabilities, let $R_{\mathcal{V}}=\sum_{v \in \mathcal{V}} R_{v}$. If $R_{\mathcal{V}}>1$, we use as final estimates $S_{v}=R_{v} / R_{\mathcal{V}}$. On the other hand, if $R_{\mathcal{V}} \leq 1$, we keep $S_{v}=R_{v}$ and introduce a dummy output $\varnothing \notin \mathcal{V}$ with estimated probability $S_{\varnothing}=1-R_{\mathcal{V}}$ (we extend the original distribution to $\mathcal{V} \cup\{\varnothing\}$, setting $p(\varnothing)=0$ ). By outputting $v$ with probability $S_{v}$, we then simulate some distribution $p^{\prime}(v)=E\left(S_{v}\right)$, and it suffices to show that $\left|E\left(S_{\mathcal{E}}\right)-p(\mathcal{E})\right| \leq$ $2 V[\beta+e(\beta)]$ for any $\mathcal{E} \subseteq \mathcal{V} \cup\{\varnothing\}$.

We first upper bound $E\left(S_{\mathcal{E}}\right)$ for $\mathcal{E} \in \mathcal{V}$. Since $S_{\mathcal{E}} \leq R_{\mathcal{E}}$, we obtain from the bounds on $R_{\mathcal{E}}$ that $\operatorname{Pr}\left[S_{\mathcal{E}} \geq\right.$ $p(\mathcal{E})+E \beta] \leq E e(\beta)$. Therefore, we have $S_{\mathcal{E}}<p(\mathcal{E})+E \beta$ with probability at least $1-E e(\beta)$, and $S_{\mathcal{E}} \leq 1$ with probability at most $E e(\beta)$. This implies that $E\left(S_{\mathcal{E}}\right) \leq p(\mathcal{E})+E[\beta+e(\beta)]$.

To lower bound $E\left(S_{\mathcal{E}}\right)$, we note that with probability at least $1-E e(\beta)$, we have $R_{\mathcal{E}}>p(\mathcal{E})-E \beta$, and with probability at least $1-V e(\beta)$, we have $R_{\mathcal{V}}<1+V \beta$. Therefore, with probability at least $1-(E+V) e(\beta)$, both these events happen at the same time, so that $S_{\mathcal{E}}=R_{\mathcal{E}} / R_{\mathcal{V}}>(p(\mathcal{E})-E \beta)(1-V \beta) \geq p(\mathcal{E})-(E+V) \beta$. This implies that $E\left(S_{\mathcal{E}}\right) \geq p(\mathcal{E})-(E+V)[\beta+e(\beta)]$. Since $S_{\varnothing}=1-S_{\mathcal{V}}$, this also implies that $E\left(S_{\varnothing}\right) \leq 2 V[\beta+e(\beta)]$.

Proof of Theorem 26 1. Let $\Lambda=\tilde{\nu}(\mathbf{p}), \mathbf{p}=q_{+} \mathbf{p}^{+}-q_{-} \mathbf{p}^{-}$, with $q_{+}, q_{-} \geq 0, q_{+}+q_{-}=\Lambda$ and $\mathbf{p}^{+}, \mathbf{p}^{-} \in \mathcal{L}$. Let $P^{+}, P^{-}$be protocols for $\mathbf{p}^{+}$and $\mathbf{p}^{-}$, respectively. These protocols use shared randomness but no communication.

To simulate $\mathbf{p}$, Alice and Bob make $T$ independent runs of $P^{+}$, where we label the outcome of the $t$-th run $\left(a_{t}^{+}, b_{t}^{+}\right)$. Similarly, let $\left(a_{t}^{-}, b_{t}^{-}\right)$be the outcome of the $t$-th run of $P^{-}$. They send the list of outcomes to the referee.

The idea is for the referee to estimate $p(a, b \mid x, y)$ based on the $2 T$ samples, and output according to the estimated distribution. Let $P_{t, a, b}^{+}$be an indicator variable which equals 1 if $a_{t}^{+}=a$ and $b_{t}^{+}=b$, and 0 otherwise. Define $P_{t, a, b}^{-}$ similarly. Furthermore, let $P_{t, a, b}=q_{+} P_{t, a, b}^{+}-q_{-} P_{t, a, b}^{-}$. Then $E\left(P_{t, a, b}\right)=p(a, b \mid x, y)$ and $P_{t, a, b} \in\left[-q_{-}, q_{+}\right]$. 
Let $P_{a, b}=\frac{1}{T} \sum_{t=1}^{T} P_{t, a, b}$ be the referee's estimate for $p(a, b \mid x, y)$. By Hoeffding's inequality,

$$
\begin{aligned}
& \operatorname{Pr}\left[P_{a, b} \geq p(a, b \mid x, y)+\beta\right] \leq e^{-\frac{2 T \beta^{2}}{\Lambda^{2}}}, \\
& \operatorname{Pr}\left[P_{a, b} \leq p(a, b \mid x, y)-\beta\right] \leq e^{-\frac{2 T \beta^{2}}{\Lambda^{2}}} .
\end{aligned}
$$

Lemma 28 with $\mathcal{V}=\mathcal{A} \times \mathcal{B}, Q_{a, b}=P_{a, b}$ and $e(\beta)=e^{-\frac{2 T \beta^{2}}{\Lambda^{2}}}$ then implies that the referee may simulate a probability distribution $\mathbf{p}^{\prime}$ such that $\delta\left(\mathbf{p}^{\prime}, \mathbf{p}\right) \leq 2 A B\left(\beta+e^{-\frac{2 T \beta^{2}}{\Lambda^{2}}}\right)$. It then suffices to set $\beta=\frac{\delta}{4 A B}$, and $T=$ $8\left[\frac{A B \Lambda}{\delta}\right]^{2} \ln \left[\frac{4 A B}{\delta}\right]$ to conclude the proof, since Alice sends $2 T \log A$ and Bob sends $2 T \log B$ bits to the referee.

For $\tilde{\nu}^{\epsilon}$, apply this proof to the distribution $\mathbf{p}^{\prime \prime}$ with statistical distance $\delta\left(\mathbf{p}, \mathbf{p}^{\prime \prime}\right) \leq \epsilon$ and $\tilde{\nu}\left(\mathbf{p}^{\prime \prime}\right)=\tilde{\nu}^{\epsilon}(\mathbf{p})$.

Note that the same proof gives an upper bound on $R_{\epsilon+\delta}^{\|, \text {ent }}$ in terms of $\tilde{\gamma}_{2}$.

2. If shared randomness is not available but quantum messages are, then we can use quantum fingerprinting [BCWd01, Yao03] to send the results of the repeated protocol to the referee. Let $\left(a^{+}(r), b^{+}(r)\right)$ be the outcomes of $P^{+}$using $r$ as shared randomness. We use the random variable $A_{a}^{+}(r)$ as an indicator variable for $a^{+}(r)=a$; similarly $B_{b}^{+}$, and $P_{\mathcal{E}}^{+}=\sum_{(a, b) \in \mathcal{E}} A_{a}^{+} B_{b}^{+}$.

We can easily adapt the proof of Newman's Theorem [New91], to show that there exists a set of $L$ random strings $\mathcal{R}=\left\{r_{1}, \ldots r_{L}\right\}$ such that $\forall x, y,\left|E_{r_{i} \in \mathcal{R}}\left(\tilde{P}_{\mathcal{E}}^{+}\left(r_{i}\right)\right)-E\left(P_{\mathcal{E}}^{+}\right)\right| \leq \alpha$ provided $L \geq \frac{4 n}{\alpha^{2}}$, where $n$ is the input length, and $\tilde{P}_{\mathcal{E}}^{+}$is the random variable where randomness is taken from $\mathcal{R}$. In other words, by taking the randomness from $\mathcal{R}$, we may simulate a probability distribution $\tilde{\mathbf{p}}^{+}$such that $\delta\left(\tilde{\mathbf{p}}^{+}, \mathbf{p}^{+}\right) \leq \alpha$.

For each $a, b \in \mathcal{A} \times \mathcal{B}$, Alice and Bob send $T$ copies of the states $\left|\phi_{a}^{+}\right\rangle=\frac{1}{\sqrt{L}} \sum_{1 \leq i \leq L}\left|A_{a}^{+}\left(r_{i}\right)\right\rangle|1\rangle|i\rangle$ and $\left|\phi_{b}^{+}\right\rangle=\frac{1}{\sqrt{L}} \sum_{1 \leq i \leq L}|1\rangle\left|B_{a}^{+}\left(r_{i}\right)\right\rangle|i\rangle$ to the referee. The inner product is

$$
\left\langle\phi_{a}^{+} \mid \phi_{b}^{+}\right\rangle=\frac{1}{L} \sum_{1 \leq i \leq L}\left\langle A_{a}^{+}\left(r_{i}\right) \mid 1\right\rangle\left\langle 1 \mid B_{b}^{+}\left(r_{i}\right)\right\rangle=\tilde{p}^{+}(a, b \mid x, y),
$$

where the expectation is taken over the random choices $r_{1}, \ldots r_{L}$.

The referee then uses inner product estimation [BCWd01]: for each copy, he performs a measurement on $\left|\phi_{a}^{+}\right\rangle \otimes$ $\left|\phi_{b}^{+}\right\rangle$to obtain a random variable $Z_{t, a, b}^{+} \in\{0,1\}$ such that $\operatorname{Pr}\left[Z_{t, a, b}^{+}=1\right]=\frac{1-\left\langle\phi_{b}^{+} \mid \phi_{a}^{+}\right\rangle^{2}}{2}$, then he sets $Z_{a, b}^{+}=$ $\frac{1}{T} \sum_{t=1}^{T} Z_{t, a, b}^{+}$. Let $Q_{a, b}^{+}=\sqrt{1-2 Z_{a, b}^{+}}$if $Z_{a, b}^{+} \leq 1 / 2$ and $Q_{a, b}^{+}=0$ otherwise. This serves as an approximation for $\tilde{p}^{+}(a, b \mid x, y)=\left|\left\langle\phi_{b}^{+} \mid \phi_{a}^{+}\right\rangle\right|$, and Hoeffding's inequality then yields

$$
\begin{aligned}
& \operatorname{Pr}\left[Q_{a, b}^{+} \geq \tilde{p}^{+}(a, b \mid x, y)+\beta\right] \leq e^{-\frac{T \beta^{4}}{2}}, \\
& \operatorname{Pr}\left[Q_{a, b}^{+} \leq \tilde{p}^{+}(a, b \mid x, y)-\beta\right] \leq e^{-\frac{T \beta^{4}}{2}} .
\end{aligned}
$$

Let $Q_{a, b}^{-}$be an estimate for $\tilde{p}^{-}(a, b \mid x, y)$ obtained using the same method. The referee then obtains an estimate for $\tilde{p}(a, b \mid x, y)=q_{+} \tilde{p}^{+}(a, b \mid x, y)-q_{-} \tilde{p}^{-}(a, b \mid x, y)$, by setting $Q_{a, b}=q_{+} Q_{a, b}^{+}+q_{-} Q_{a, b}^{-}$, such that

$$
\begin{aligned}
& \operatorname{Pr}\left[Q_{a, b} \geq \tilde{p}(a, b \mid x, y)+\beta\right] \leq 2 e^{-\frac{T \beta^{4}}{2 \Lambda^{4}}}, \\
& \operatorname{Pr}\left[Q_{a, b} \leq \tilde{p}(a, b \mid x, y)-\beta\right] \leq 2 e^{-\frac{T \beta^{4}}{2 \Lambda^{4}}} .
\end{aligned}
$$

Lemma 28 with $e(\beta)=2 e^{-\frac{T \beta^{4}}{2 \Lambda^{4}}}$ then implies that the referee may simulate a probability distribution $\mathbf{p}^{s}$ such that $\delta\left(\mathbf{p}^{s}, \tilde{\mathbf{p}}\right) \leq 2 A B\left(\beta+2 e^{-\frac{T \beta^{4}}{2 \Lambda^{4}}}\right)$. Since $\delta(\tilde{\mathbf{p}}, \mathbf{p}) \leq \Lambda \alpha$, we need to pick $T, L=\frac{4 n}{\alpha}$ large enough so that $\Lambda \alpha+$ $2 A B\left[\beta+2 e^{-T \beta^{4} / 2 \Lambda^{4}}\right] \leq \delta$. Setting $\alpha=\frac{\delta}{2 \Lambda}, \beta=\frac{\delta}{8 A B}, T=2 \frac{\Lambda^{4}}{\beta^{4}} \ln \left(\frac{16 A B}{\delta}\right)=2^{13}\left[\frac{A B \Lambda}{\delta}\right]^{4} \ln \left(\frac{16 A B}{\delta}\right)$ and $L=$ $\frac{4 n}{\alpha^{2}}=\frac{16 n \Lambda^{2}}{\delta^{2}}$, the total complexity of the protocol is $4 A B T(\log (L)+2)=O\left((A B)^{5}\left[\frac{\Lambda}{\delta}\right]^{4} \ln \left[\frac{A B}{\delta}\right] \log (n)\right)$. (We may assume that $\frac{\Lambda}{\delta} \leq n^{1 / 4}$, otherwise this protocol performs worse than the trivial protocol.) 
In the case of Boolean functions, the referee's job is made easier by the fact that he only needs to determine the sign of the correlation with probability $1-\delta$. This allows us to get some improvements in the upper bounds. Similar improvements can be obtained for other types of promises on the distribution.

Theorem 29. Let $f:\{0,1\}^{n} \times\{0,1\}^{n} \rightarrow\{0,1\}$, with associated sign matrix $C_{f}$, and $\epsilon, \delta<1 / 2$.

1. $R_{\delta}^{\|, \operatorname{pub}}(f) \leq 4\left[\frac{\tilde{\nu}^{\epsilon}\left(C_{f}\right)}{1-2 \epsilon}\right]^{2} \ln \left(\frac{1}{\delta}\right)$,

2. $Q_{\delta}^{\|}(f) \leq O\left(\log (n)\left[\frac{\tilde{\nu}^{\epsilon}\left(C_{f}\right)}{1-2 \epsilon}\right]^{4} \ln \left(\frac{1}{\delta}\right)\right)$.

From Lemmas 16 and 21, these bounds may also be expressed in terms of $\gamma_{2}^{\alpha}$, and the best upper bounds are obtained from $\gamma_{2}^{\infty}\left(C_{f}\right)=\frac{1}{\epsilon^{\text {ent }}\left(C_{f}\right)}$. The first item then coincides with the upper bound of [LS08b].

Together with the lower bounds from Section 3, the first and last items also imply the results of Yao, Shi and Zhu, and Gavinsky et al. [Yao03, SZ08, GKd06], who show how to simulate any (logarithmic) communication protocol in the simultaneous messages model, with an exponential blowup in communication. These results extend to arbitrary distributions. In particular, this gives as a special case a much simpler proof of the constant upper bound on approximating quantum distributions, which Shi and Zhu prove using sophisticated techniques based on diamond norms.

Corollary 30. Let $f:\{0,1\}^{n} \times\{0,1\}^{n} \rightarrow\{0,1\}$. If $Q_{\epsilon}^{\text {ent }}(f) \leq q$, then
1. $R_{\delta}^{\|, \text {pub }}(f) \leq K_{G}^{2} \cdot 2^{2 q+2} \ln \left(\frac{1}{\delta}\right) \frac{1}{(1-2 \epsilon)^{2}}$,
2. $Q_{\delta}^{\|}(f) \leq O\left(\log (n) 2^{4 q} \ln \left(\frac{1}{\delta}\right) \frac{1}{(1-2 \epsilon)^{4}}\right)$.

For any distribution $\mathbf{p} \in \mathcal{C}$ with inputs in $\mathcal{X} \times \mathcal{Y}$ with $|\mathcal{X} \times \mathcal{Y}| \leq 2^{n}$, and outcomes in $\mathcal{A} \times \mathcal{B}$ with $A=|\mathcal{A}|, B=|\mathcal{B}|$, and any $\epsilon, \delta<1 / 2$, if $R_{\epsilon}(\mathbf{p}) \leq c$ and $Q_{\epsilon}(\mathbf{p}) \leq q$,

1. $R_{\epsilon+\delta}^{\|, p u b}(\mathbf{p}) \leq O\left(2^{4 q} \frac{(A B)^{3}}{\delta^{2}} \ln ^{2}\left[\frac{A B}{\delta}\right]\right)$,

2. $Q_{\epsilon+\delta}^{\|}(\mathbf{p}) \leq O\left(2^{8 q} \frac{(A B)^{9}}{\delta^{4}} \ln \left[\frac{A B}{\delta}\right] \log (n)\right)$.

\section{Conclusion and open problems}

By studying communication complexity in the framework provided by the study of quantum non-locality (and beyond), we have given very natural and intuitive interpretations of the otherwise very abstract lower bounds of Linial and Shraibman. Conversely, bridging this gap has allowed us to port these very strong and mathematically elegant lower bound methods to the much more general problem of simulating non-signaling distributions.

Since many communication problems may be reduced to the task of simulating a non-signaling distribution, we hope to see applications of this lower bound method to concrete problems for which standard techniques do not apply, in particular for cases that are not Boolean functions, such as non-Boolean functions, partial functions or relations. Let us also note that our method can be generalized to multipartite non-signaling distributions, and will hopefully lead to applications in the number-on-the-forehead model, for which quantum lower bounds seem hard to prove.

In the case of binary distributions with uniform marginals (which includes in particular Boolean functions), Tsirelson's theorem (Theorem 7 ) and the existence of Grothendieck's constant (Proposition 9) imply that there is at most a constant gap between $\nu$ and $\gamma_{2}$. For this reason, it was known that Linial and Shraibman's factorization norm lower bound technique give lower bounds of the same of order for classical and quantum communication (note that this is also true for the related discrepancy method). Despite the fact that Tsirelson's theorem and Grothendieck's inequality are not known to extend beyond the case of Boolean outcomes with uniform marginals, we have shown that in the general case of distributions, there is also a constant gap between $\tilde{\nu}$ and $\tilde{\gamma}_{2}$. While this may be seen as a negative result, this also reveals interesting information about the structure of the sets of local and quantum distributions. In particular, this could have interesting consequences for the study of non-local games. 


\section{Acknowledgements}

We are grateful to Benjamin Toner for pointing us towards the existing literature on non-signaling distributions as well as very useful discussions of the Linial and Shraibman lower bound on communication complexity. We also thank Peter Høyer, Troy Lee, Oded Regev, Mario Szegedy, and Dieter van Melkebeek with whom we had many stimulating discussions. Part of this work was done while J. Roland was affiliated with FNRS Belgium and U.C. Berkeley. The research was supported by the EU 5th framework program QAP, and by the ANR Blanc AlgoQP.

\section{References}

[AN06] Noga Alon and Assaf Naor. Approximating the cut-norm via Grothendieck's inequality. SIAM J. Comput., 35(4):787-803, 2006.

[Bar07] Jonathan Barrett. Information processing in generalized probabilistic theories. Physical Review A, 75(3):032304, 2007.

[BBLW07] Howard Barnum, Jonathan Barrett, Matthew Leifer, and Alexander Wilce. Generalized no-broadcasting theorem. Physical Review Letters, 99(240501), 2007.

[BCT99] Gilles Brassard, Richard Cleve, and Alain Tapp. Cost of Exactly Simulating Quantum Entanglement with Classical Communication. Physical Review Letters, 83:1874-1877, 1999. quant-ph/9901035.

[BCW98] Harry Buhrman, Richard Cleve, and Avi Wigderson. Quantum vs. classical communication and computation. In Proceedings of the thirtieth annual ACM symposium on Theory of computing, pages 63-68, 1998.

[BCWd01] Harry Buhrman, Richard Cleve, John Watrous, and Ronald de Wolf. Quantum fingerprinting, 2001.

[Bel64] John S. Bell. On the Einstein Podolsky Rosen paradox. Physics, 1:195, 1964.

$\left[\mathrm{BLM}^{+}\right.$05] Jonathan Barrett, Noah Linden, Serge Massar, Stefano Pironio, Sandu Popescu, and David Roberts. Nonlocal correlations as an information theoretic resource. Physical Review A, 71:022101, 2005.

[BP05] Jonathan Barrett and Stefano Pironio. Popescu-Rohrlich correlations as a unit of nonlocality. Physical Review Letters, 95:140401, 2005.

[CGMP05] Nicolas J. Cerf, Nicolas Gisin, Serge Massar, and Sandu Popescu. Simulating Maximal Quantum Entanglement without Communication. Physical Review Letters, 94(22):220403, 2005.

[DLR07] Julien Degorre, Sophie Laplante, and Jérémie Roland. Classical simulation of traceless binary observables on any bipartite quantum state. Physical Review A, 75(012309), 2007.

[Fey86] Richard Feynman. Negative probability. In B. J. Hiley and F. David Peat, editors, Quantum Implications: Essays in Honor of David Bohm, pages 235-248, London, 1986. Routledge and Kegan Paul.

[FR81] David J. Foulis and Charles H. Randall. Empirical logic and tensor products. In Interpretations and Foundations of Quantum Theory, volume Interpretations and Foundations of Quantum Theory, pages 120. Wissenschaftsverlag, BibliographischesInstitut, 1981.

[Gav08] Dmitry Gavinsky. Classical interaction cannot replace quantum non-locality. 2008. In preparation.

[GKd06] Dmitry Gavinsky, Julia Kempe, and Ronald de Wolf. Strengths and weaknesses of quantum fingerprinting. In Proceedings of the 21 st Conference on Computational Complexity, pages 288-295, 2006.

[Gro85] H.J. Groenewold. The elusive quantal individual. Physics Reports, 127(6):379-401, 1985. 
[JKN08] Rahul Jain, Hartmut Klauck, and Ashwin Nayak. Direct product theorems for communication complexity via subdistribution bounds. In Proceedings of the Fortieth Annual ACM Symposium on the Theory of Computing, 2008.

[JM05] Nick S. Jones and Lluis Masanes. Interconversion of nonlocal correlations. Physical Review A, 72:052312, 2005 .

[KN97] Eyal Kushilevitz and Noam Nisan. Communication complexity. Cambridge University Press, New York, 1997.

[Kre95] Ilan Kremer. Quantum communication. Master's thesis, The Hebrew University of Jerusalem, 1995.

[KRF87] Matthias Kläy, Charles H. Randall, and David J. Foulis. Tensor products and probability weights. International Journal of Theoretical Physics, 26(3):199-219, 1987.

[LMSS07] Nati Linial, Shahar Mendelson, Gideon Schechtman, and Adi Shraibman. Complexity measures of sign matrices. Combinatorica, 27:439-463, 2007.

[LS08a] Troy Lee and Adi Shraibman. Disjointness is hard in the multi-party number on the forehead model. In Proceedings of the 23rd Conference on Computational Complexity, 2008. To appear.

[LS08b] Nati Linial and Adi Shraibman. Lower bounds in communication complexity based on factorization norms. Random Structures and Algorithms, 2008. To appear.

[LSŠ08] Troy Lee, Adi Shraibman, and Robert Špalek. A direct product theorem for discrepancy. In Proceedings of the 21rd Conference on Computational Complexity, 2008. To appear.

[Mau92] Tim Maudlin. Bell's inequality, information transmission, and prism models. In Biennal Meeting of the Philosophy of Science Association, pages 404-417, 1992.

[McD91] Colin McDiarmid. Concentration. In Probabilistic Methods for Algorithmic Discrete Mathematics. Springer, 1991.

[New91] Ilan Newman. Public vs. private coin flips in one round communication games. Information Processing Letters, 39(2):67-71, 1991.

[NPA08] Miguel Navascues, Stefano Pironio, and Antonio Acin. A convergent hierarchy of semidefinite programs characterizing the set of quantum correlations. Technical Report arXiv.org:0803.4290, arXiv ePrint archive, 2008.

[Pir03] Stefano Pironio. Violations of Bell inequalities as lower bounds on the communication cost of nonlocal correlations. Physical Review A, 68(6):062102, 2003.

[RF81] Charles H. Randall and David J. Foulis. Operational statistics and tensor products. In Interpretations and Foundations of Quantum Theory, volume Interpretations and Foundations of Quantum Theory, pages 21-28. Wissenschaftsverlag, BibliographischesInstitut, 1981.

[RT07] Oded Regev and Benjamin Toner. Simulating quantum correlations with finite communication. In Proceedings of 48th Annual IEEE Symposium on Foundations of Computer Science, pages 384-394, 2007.

[Ste00] Michael Steiner. Towards quantifying non-local information transfer: finite-bit non-locality. Phys. Letters A, 270:239-244, 2000.

[SZ08] Yaoyun Shi and Yufan Zhu. Tensor norms and the classical communication complexity of bipartite quantum measurements. SIAM Journal on Computing, 2008. To appear.

[TB03] Benjamin F. Toner and Dave Bacon. Communication Cost of Simulating Bell Correlations. Physical Review Letters, 91:187904, 2003. 
[Tsi80] Boris S. Tsirelson. Quantum generalizations of Bell's inequality. Letters Math. Phys., 4(2):93-100, 1980.

[Tsi85] Boris S. Tsirelson. Problems of the theory probability distributions ix. Zapiski Math. Inst. Steklov (LOMI), 142:174-194, 1985. English translation in Quantum analogues of the Bell inequalities. The case of two spatially separated domains, J. Soviet Math. 36, 557-570 (1987).

[VB96] Lieven Vandenberghe and Stephen Boyd. Semidefinite programming. SIAM Review, 38(1):49-95, 1996.

[vN28] John von Neumann. Zur Theorie der Gesellschaftsspiele. Mathematische Annalen, 100(1):295-320, 1928.

[Wi192] Andrew Wilce. Tensor products in generalized measure theory. International Journal of Theoretical Physics, 31(11):1915-1928, 1992.

[Yao79] Andrew Chi-Chih Yao. Some complexity questions related to distributive computing. In Proceedings of the eleventh annual ACM symposium on Theory of computing, pages 209-213, 1979.

[Yao93] Andrew Chi-Chih Yao. Quantum circuit complexity. In Proceedings of the 34th Annual Symposium on Foundations of Computer Science, pages 352-361, 1993.

[Yao03] Andrew Chi-Chih Yao. On the power of quantum fingerprinting. In Proceedings of the thirty-fifth annual ACM symposium on Theory of Computing, pages 77-81, 2003.

\section{A Proof of Lemma 11}

The proof relies on the following observation:

Claim 1. Let $\left|\psi_{t}\right\rangle$ be the entangled state shared by Alice and Bob after the first $t=t_{A}+t_{B}$ qubits of communication ( $t_{A}$ bits from Alice to Bob, and $t_{B}$ bits from Bob to Alice). This state may be written as $\left|\psi_{t}\right\rangle=$ $\sum_{i \in I} \mu_{i} \sum_{T \in\{0,1\}^{t}} A_{T}\left|\alpha^{(i)}\right\rangle B_{T}\left|\beta^{(i)}\right\rangle$, where $\sum_{i}\left|\mu_{i}\right|^{2}=1,\left\{\left|\alpha^{(i)}\right\rangle: \forall i \in I\right\}$ and $\left\{\left|\beta^{(i)}: \forall i \in I\right\rangle\right\}$ are orthonormal bases for Alice and Bob's initial registers respectively and $A_{T}, B_{T}$ are linear operators such that:

- $A_{0}, B_{0}$ are the identity operators on Alice and Bob's initial registers, respectively,

- $A_{T}$ are linear operators acting on Alice's initial register and depending on her input only, satisfying $\sum_{T \in\{0,1\}^{t}} \| A_{T}\left|\psi_{A}\right\rangle \|^{2}=$ $2^{t_{B}}$ for all (unit) state $\left|\psi_{A}\right\rangle$ of Alice's register.

- $B_{T}$ are linear operators depending on Bob's input only, satisfying $\sum_{T \in\{0,1\}^{t}} \| B_{T}\left|\psi_{B}\right\rangle \|^{2}=2^{t_{A}}$ for all (unit) state $\left|\psi_{B}\right\rangle$ of Bob's register.

Proof of Claim 1. We prove this by induction over $t$. This is true for $t=0$, since using Schmidt decomposition, we may write the initial entangled state shared by Alice and Bob, before the quantum communication protocol is initiated, as $\left|\psi_{0}\right\rangle=\sum_{i \in I} \mu_{i}\left|\alpha^{(i)}\right\rangle\left|\beta^{(i)}\right\rangle$, where $\sum_{i}\left|\mu_{i}\right|^{2}=1$ and $\left\{\left|\alpha^{(i)}\right\rangle: \forall i \in I\right\}$ and $\left\{\left|\beta^{(i)}: \forall i \in I\right\rangle\right\}$ are orthonormal bases for Alice and Bob's registers respectively (as is, these are actually just orthonormal, but we can always obtain a basis by setting $\mu_{i}=0$ for the missing basis vectors).

If this is true for $t-1$, then we have $\left|\psi_{t-1}\right\rangle=\sum_{i \in I} \mu_{i} \sum_{T \in\{0,1\}^{t-1}} A_{T}\left|\alpha^{(i)}\right\rangle B_{T}\left|\beta^{(i)}\right\rangle$, where $\sum_{T \in\{0,1\}^{t-1}} \| A_{T}\left|\alpha^{(i)}\right\rangle \|^{2}=2^{t_{B}}$ and $\sum_{T \in\{0,1\}^{t-1}} \| B_{T}\left|\beta^{(i)}\right\rangle \|^{2}=2^{t_{A}-1}$ for all $i \in I$ (we assume wlog that the $t$ 's qubit is sent by Alice to Bob). Alice's operation at turn $t$ will be to apply some unitary operation $U_{t}$ on her register, then send one of the qubits in her register to Bob. By isolating this qubit, we define the linear operators $A_{T 0}$ and $A_{T 1}$ to be such that $U_{t} A_{T}\left|\alpha^{(i)}\right\rangle=A_{T 0}\left|\alpha^{(i)}\right\rangle|0\rangle+A_{T 1}\left|\alpha^{(i)}\right\rangle|1\rangle$ for all $i \in I$. Unitarity then implies that $\| A_{T 0}\left|\alpha^{(i)}\right\rangle\left\|^{2}+\right\| A_{T 1}\left|\alpha^{(i)}\right\rangle\left\|^{2}=\right\| A_{T}\left|\alpha^{(i)}\right\rangle \|^{2}$, and as a consequence $\sum_{T \in\{0,1\}^{t}} \| A_{T}\left|\alpha^{(i)}\right\rangle \|^{2}=2^{t_{B}}$. We then have

$$
\begin{aligned}
\left|\psi_{t}\right\rangle & =\sum_{i \in I} \mu_{i} \sum_{T \in\{0,1\}^{t-1}}\left[A_{T 0}\left|\alpha^{(i)}\right\rangle|0\rangle B_{T}\left|\beta^{(i)}\right\rangle+A_{T 1}\left|\alpha^{(i)}\right\rangle|1\rangle B_{T}\left|\beta^{(i)}\right\rangle\right] \\
& =\sum_{i \in I} \mu_{i} \sum_{T \in\{0,1\}^{t}} A_{T}\left|\alpha^{(i)}\right\rangle B_{T}\left|\beta^{(i)}\right\rangle
\end{aligned}
$$


where, for all $T \in\{0,1\}^{t-1}$, we have defined linear operators $B_{T 0}, B_{T 1}$ such that $B_{T 0}\left|\beta^{(i)}\right\rangle=|0\rangle B_{T}\left|\beta^{(i)}\right\rangle$ and $B_{T 1}\left|\beta^{(i)}\right\rangle=|1\rangle B_{T}\left|\beta^{(i)}\right\rangle$ for all $i \in I$, considering that the additional qubit is in Bob's hands at the end of turn $t$. Furthermore, we have $\| B_{T 0}\left|\beta^{(i)}\right\rangle\left\|^{2}+\right\| B_{T 1}\left|\beta^{(i)}\right\rangle\left\|^{2}=2\right\| B_{T}\left|\beta^{(i)}\right\rangle \|^{2}$, and as a consequence $\sum_{T \in\{0,1\}^{t}} \| B_{T}\left|\beta^{(i)}\right\rangle \|^{2}=$ $2^{t_{A}}$, which completes the proof of our claim.

Proof of Lemma 11] At the end of the quantum communication protocol, Alice and Bob share a quantum state $\left|\psi_{q}\right\rangle$ satisfying Claim 1 for $t=q$. Alice and Bob then perform binary $(\{+1,-1\}$-valued) measurements $A$ and $B$ on their respective parts of the state. By orthonormality of the states $\left|\psi_{q}^{(i)}\right\rangle$, we have for the correlation

$$
\begin{aligned}
C & =\left\langle\psi_{q}|A B| \psi_{q}\right\rangle \\
& =\sum_{i, j \in I} \mu_{i}^{*} \mu_{j} \sum_{T, U \in\{0,1\}^{q}}\left\langle\alpha^{(i)}\left|A_{T}^{\dagger} A A_{U}\right| \alpha^{(j)}\right\rangle\left\langle\beta^{(i)}\left|B_{T}^{\dagger} B B_{U}\right| \beta^{(j)}\right\rangle .
\end{aligned}
$$

We may now define the vectors $\vec{a}(x)$ and $\vec{b}(y)$ in a $2^{2 t}|I|^{2}$-dimensional complex vector space, with coordinates

$$
\begin{aligned}
& a_{T U i j}(x)=\mu_{i}\left\langle\alpha^{(j)}\left|A_{U}^{\dagger} A A_{T}\right| \alpha^{(i)}\right\rangle, \\
& b_{T U i j}(x)=\mu_{j}\left\langle\beta^{(i)}\left|B_{T}^{\dagger} B B_{U}\right| \beta^{(j)}\right\rangle, \quad \forall T, U \in\{0,1\}^{q}, i, j \in I,
\end{aligned}
$$

so that $C=\vec{a}(x) \cdot \vec{b}(y)$. Moreover, using the fact that the $\left|\alpha^{(j)}\right\rangle$ 's define an orthonormal basis for Alice's register and the property on the norms of the operators $A_{T}$, we have

$$
\begin{aligned}
\|\vec{a}(x)\|^{2} & =\sum_{i, j \in I}\left|\mu_{i}\right|^{2} \sum_{T, U \in\{0,1\}^{q}}\left|\left\langle\alpha^{(j)}\left|A_{U}^{\dagger} A A_{T}\right| \alpha^{(i)}\right\rangle\right|^{2} \\
& =\sum_{i \in I}\left|\mu_{i}\right|^{2} \sum_{T, U \in\{0,1\}^{q}} \| A_{U}^{\dagger} A A_{T}\left|\alpha^{(i)}\right\rangle \|^{2} \\
& \leq \sum_{i \in I}\left|\mu_{i}\right|^{2} \sum_{T, U \in\{0,1\}^{q}} \| A_{U}^{\dagger}\left|\phi_{T}^{(i)}\right\rangle\left\|^{2}\right\| A_{T}\left|\alpha^{(i)}\right\rangle \|^{2}=2^{2 q_{B}},
\end{aligned}
$$

where $\left|\phi_{T}^{(i)}\right\rangle$ is the renormalized state $A A_{T}\left|\alpha^{(i)}\right\rangle$. So, we have $\|\vec{a}(x)\| \leq 2^{q_{B}}$, and similarly $\|\vec{b}(y)\| \leq 2^{q_{A}}$.

\section{B Proof of Theorem 13, Boolean case}

We show that $\tilde{\nu}(\mathbf{p}) \leq 2^{R_{0}(\mathbf{p})}$ for a distribution $\mathbf{p}$ over $\mathcal{A} \times \mathcal{B}$ with $|\mathcal{A}|=A$ and $|\mathcal{B}|=B$. The claim follows by lettin $A=B=2$. We give a proof for the classical case, and the quantum case follows by using teleportation. Let $c_{A}$ be the number of bits sent from Alice to Bob and $c_{B}$ from Bob to Alice. From Theorem 10 , we know that $\mathbf{p}_{\pi_{A}, \pi_{B}}$ is local for $\pi_{A}=2^{-c_{A}}, \pi_{B}=2^{-c_{B}}$. Therefore, there exists a protocol $P^{\prime}$ for $\mathbf{p}_{\pi_{A}, \pi_{B}}$, using shared randomness but no communication. From this protocol, we define four protocols $P^{++}, P^{+-}, P^{-+}, P^{--}$simulating four different probability distributions $\mathbf{p}_{++}, \mathbf{p}_{+-}, \mathbf{p}_{-+}, \mathbf{p}_{--}$. Each protocol starts exactly as $P^{\prime}$ until the players produce their output. At this point, they may substitute the output from $P^{\prime}$ by another output

1. In $P^{++}$, the players output as in $P^{\prime}$

2. In $P^{+-}$, Alice outputs as in $P^{\prime}$, but Bob substitutes his output by one of the other $B-1$ possible outputs, chosen uniformly at random,

3. In $P^{-+}$, Bob outputs as in $P^{\prime}$, but Alice substitutes her output by one of the other $A-1$ possible outputs, chosen uniformly at random,

4. In $P^{--}$, both Alice and Bob substitute their outputs by one of the other possible outputs, chosen uniformly at random. 
It is straightforward to check that these protocols simulate the following (local) non-signaling distributions:

$$
\begin{aligned}
p^{++}(a, b \mid x, y) & =p_{\pi_{A}, \pi_{B}}(a, b \mid x, y), \\
p^{+-}(a, b \mid x, y) & =\frac{1}{B-1}\left[p_{\pi_{A}, \pi_{B}}(a \mid x)-p_{\pi_{A}, \pi_{B}}(a, b \mid x, y)\right], \\
p^{-+}(a, b \mid x, y) & =\frac{1}{A-1}\left[p_{\pi_{A}, \pi_{B}}(b \mid y)-p_{\pi_{A}, \pi_{B}}(a, b \mid x, y)\right], \\
p^{--}(a, b \mid x, y) & =\frac{1}{(A-1)(B-1)}\left[1-p_{\pi_{A}, \pi_{B}}(a \mid x)-p_{\pi_{A}, \pi_{B}}(b \mid y)+p_{\pi_{A}, \pi_{B}}(a, b \mid x, y)\right] .
\end{aligned}
$$

Let us define the following quasi-probabilities:

$$
\begin{aligned}
q_{++} & =\frac{1}{A B \pi}\left(A-1+\pi_{A}\right)\left(B-1+\pi_{B}\right), \\
q_{+-} & =-\frac{1}{A B \pi}\left(A-1+\pi_{A}\right)(B-1)\left(1-\pi_{B}\right), \\
q_{-+} & =-\frac{1}{A B \pi}(A-1)\left(1-\pi_{A}\right)\left(B-1+\pi_{B}\right), \\
q_{--} & =\frac{1}{A B \pi}(A-1)(B-1)\left(1-\pi_{A}\right)\left(1-\pi_{B}\right) .
\end{aligned}
$$

These satisfy

$$
\begin{aligned}
& q_{++} p^{++}(a, b \mid x, y)+q_{+-} p^{+-}(a, b \mid x, y)+q_{-+} p^{-+}(a, b \mid x, y)+q_{--} p^{--}(a, b \mid x, y) \\
& \quad=\frac{1}{\pi} p_{\pi_{A}, \pi_{B}}(a, b \mid x, y)+\frac{1}{\pi_{A}}\left(1-\frac{1}{\pi_{B}}\right) p_{\pi_{A}, \pi_{B}}(a \mid x) \frac{1}{B}+\left(1-\frac{1}{\pi_{A}}\right) \frac{1}{\pi_{B}} \frac{1}{A} p_{\pi_{A}, \pi_{B}}(b \mid y)+\left(1-\frac{1}{\pi_{A}}\right)\left(1-\frac{1}{\pi_{B}}\right) \frac{1}{A B} \\
& \quad=p(a, b \mid x, y)
\end{aligned}
$$

so this determines an affine model for $p(a, b \mid x, y)$. Moreover, we have

$$
\left|q_{++}\right|+\left|q_{+-}\right|+\left|q_{-+}\right|+\left|q_{--}\right|=\frac{1}{A B \pi}\left[2(A-1)-(A-2) \pi_{A}\right]\left[2(B-1)-(B-2) \pi_{B}\right] .
$$

When $A=B=2$ this equals $\frac{1}{\pi}$. 